\title{
THE RISE AND FALL OF V4334 SAGITTARII (SAKURAI'S OBJECT)
}

\author{
H. W. Duerbeck, ${ }^{1}$ W. Liller, ${ }^{2}$ C. Sterken, ${ }^{1}$ S. Benetti, ${ }^{3}$ A. M. van Genderen,,${ }^{4}$ J. Arts, ${ }^{4}$ J. D. Kurk,,${ }^{4}$ M. Janson, ${ }^{5}$ \\ T. Voskes, ${ }^{4}$ E. Brogt, ${ }^{5}$ T. Arentoft, ${ }^{1}$ A. Van Der Meer, ${ }^{4}$ AND R. Dijkstra ${ }^{5}$ \\ Received 1999 October 21 ; accepted 2000 January 31
}

\begin{abstract}
CCD UBVRi photometry of the final helium flash object V4334 Sgr (Sakurai's object) carried out during 1997-1999 is presented, and the light curve from its prediscovery rise to the dust obscuration phase is constructed. The optical light curve can be divided into four sections, the rise to maximum, the maximum, the dust onset, and the massive dust shell phase. The color indices show a general increase with time, first because of the photospheric expansion and cooling and later because of the dust-forming events. The energy distributions for the years 1996-1999 show that an increasing part of the energy is radiated at infrared wavelengths. In 1996 the infrared excess is likely caused by free-free radiation in the stellar wind. Starting from 1997 or 1998 at the latest, carbon dust grains are responsible for the more and more dramatic decrease of optical radiation and the growing infrared excess. Its photometric behavior in 1998-1999 mimics the "red declines" of R CrB variables; the amplitude, however, is more extreme than any fading ever observed in an R CrB star. Evidence is given that a complete dust shell has formed around V4334 Sgr. It therefore shows similarities with dust-forming classical novae, although it is evolving $\sim 20$ times more slowly. Its luminosity increased by a factor 4 between 1996 and 1998. A comparison of timescales of the final helium flash objects FG Sge, V605 Aql, and V4334 Sgr shows that the observed photometric and spectroscopic features are similar, while V4334 Sgr is the most rapidly evolving object to date.
\end{abstract}

Key words: stars: AGB and post-AGB — stars: individual (V4334 Sagittarii) - stars: variables: other

\section{INTRODUCTION}

After completing their core helium-burning phase, stars less massive than $\sim 10.5 M_{\odot}$ develop in their AGB phase electron-degenerate cores of carbon and oxygen (or oxygen and neon at the massive end) and alternately burn helium or hydrogen in shells. Each quiescent helium-burning phase is preceded by a thermonuclear runaway in the degenerate helium layer. In the aftermath of each of these "thermal pulses," carbon and s-process elements are transported to the stellar surface (Iben \& MacDonald 1995). Finally, these stars undergo extensive mass loss ("superwind phase") and move in the Hertzsprung-Russell diagram to the region of central stars of planetary nebulae (PNN).

The peculiar variable star FG Sge, which is situated in the center of a planetary nebula, has inspired theoreticians to study the post-AGB evolution in more detail. It is now believed that in about $10 \%$ of all thermally pulsing stars, the final pulse can occur in a very late stage, when the star has already settled down as a PNN. In such a case, the final pulse is directly observable as a "final He flash," which drives the star from the region of the PNN back to the top of the AGB. This happened to FG Sge in the course of the twentieth century. Such a "born-again giant" phase can last decades, centuries, or millennia, but the object will

\footnotetext{
${ }^{1}$ WE/OBSS, Free University Brussels (VUB), Pleinlaan 2, B-1050 Brussels, Belgium; hduerbec@vub.ac.be, csterken@vub.ac.be, tarentof@vub.ac.be.

${ }^{2}$ Instituto Isaac Newton, Ministerio de Educación de Chile, Correo 9, Casilla 8-9, Santiago, Chile; wliller@compuserve.com.

${ }^{3}$ TGN, Centro Galileo Galilei, Calle Alvarez de Abreu, 70, E-38700 Santa Cruz de La Palma, Canary Islands, Spain; benetti@tng.iac.es.

${ }^{4}$ Leiden Observatory, Postbus 9513, NL-2300 RA Leiden, Netherlands; genderen@strw.LeidenUniv.nl.

${ }^{5}$ Kapteyn Institute, University of Groningen, P.O. Box 800, NL-9700 AV Groningen, Netherlands.
}

return finally to the PNN region. During the final He flash phase, the outer layers of the star undergo extensive nucleosynthesis, including more or less complete processing of the surficial hydrogen. A large fraction of the outer layers are ejected, leading to the formation of a hydrogen-poor, carbon-rich nebulosity in the center of the planetary nebula. Planetary nebulae with central hydrogen-poor condensations such as Abell 30 or Abell 80 (Jacoby 1979; Jacoby \& Ford 1983), H-deficient post-AGB stars such as WolfRayet-type central stars of planetary nebulae, or white dwarfs of the PG 1159 type (e.g., Werner et al. 1999) are possible end products of final He flash objects.

The evolution of a final He flash from the PNN to the giant stage may take decades (as in FG Sge) or only a few years, as concluded in recent years from the present state and a few historical observations of "Nova Aquilae No. 4" of 1919, also known as V605 Aql (Seitter 1985; Clayton \& De Marco 1997). Seventy-seven years after the flareup of V605 Aql, a "novalike object in Sagittarius" was soon recognized as another one of these rare events. It offers the first opportunity to study in detail the evolution of a fast final He flash.

Sakurai's object, later named V4334 Sgr, was discovered on 1996 February 20 as a star of $11^{\text {th }}$ magnitude by Y. Sakurai (Nakano, Benetti, \& Duerbeck 1996). Prediscovery observations by Y. Sakurai and K. Takamizawa showed that it had been at magnitude 12.5 in early 1995 and possibly at magnitude 15.5 in late 1994 . Several groups have monitored the optical brightness evolution: a Russian group (Arkhipova \& Noskova 1997; Arkhipova et al. 1998, 1999), a US group using automatic photometric telescopes (Margheim, Guinan, \& McCook 1997; Guinan et al. 1998), and a Chilean-European group, whose results are presented here. Furthermore, scattered observations made with larger telescopes during interesting phases of its evolution will also be discussed. 
TABLE 1

UBVRi ObSERVATIONS OF V4334 SAgItTARII

\begin{tabular}{|c|c|c|c|c|c|c|}
\hline $\operatorname{HJD}(2,450,000+)$ & $U$ & $B$ & $V$ & $R_{\mathrm{C}}$ & $i_{\mathrm{G}}$ & Observer \\
\hline $504.884 \ldots \ldots \ldots \ldots$ & & & 10.95 & & & WL \\
\hline $514.867 \ldots \ldots \ldots \ldots$ & & & 11.05 & & 9.18 & TV \\
\hline $515.877 \ldots \ldots \ldots \ldots$ & & & 11.04 & & 9.18 & $\mathrm{TV}$ \\
\hline $516.889 \ldots \ldots \ldots \ldots$ & & & 11.04 & & 9.18 & TV \\
\hline $516.913 \ldots \ldots \ldots \ldots$ & & & 11.04 & & & WL \\
\hline $517.882 \ldots \ldots \ldots \ldots$ & & & 11.04 & & 9.19 & TV \\
\hline $518.875 \ldots \ldots \ldots \ldots$ & & & 11.04 & & 9.19 & TV \\
\hline $519.889 \ldots \ldots \ldots \ldots$ & & & 11.04 & & 9.20 & TV \\
\hline $520.901 \ldots \ldots \ldots \ldots$ & & & 11.02 & & 9.20 & TV \\
\hline $521.907 \ldots \ldots \ldots \ldots$ & & & 11.02 & & 9.18 & TV \\
\hline $521.906 \ldots \ldots \ldots \ldots$ & & & 10.99 & & & WL \\
\hline $522.884 \ldots \ldots \ldots \ldots$ & & & 11.02 & 10.01 & 9.18 & TV \\
\hline $523.861 \ldots \ldots \ldots \ldots$ & & & 11.02 & & 9.18 & TV \\
\hline $539.914 \ldots \ldots \ldots \ldots$ & 13.49 & 12.60 & 10.92 & 9.93 & 8.96 & MT \\
\hline $555.896 \ldots \ldots \ldots \ldots$ & 13.32 & 12.46 & 10.86 & 9.82 & 8.94 & SB \\
\hline $556.924 \ldots \ldots \ldots \ldots$ & 13.37 & 12.50 & 10.86 & 9.86 & 8.94 & SB \\
\hline $557.728 \ldots \ldots \ldots \ldots$ & & & 10.82 & & & WL \\
\hline $563.769 \ldots \ldots \ldots \ldots$ & & & 10.95 & & & WL \\
\hline $570.701 \ldots \ldots \ldots \ldots$ & & & 11.10 & & & WL \\
\hline $574.710 \ldots \ldots \ldots \ldots$ & & & 11.12 & & & WL \\
\hline $581.679 \ldots \ldots \ldots \ldots$ & & & 11.06 & & & WL \\
\hline $587.803 \ldots \ldots \ldots \ldots$ & & & 11.09 & & & WL \\
\hline $590.705 \ldots \ldots \ldots \ldots$ & & & 11.03 & & & WL \\
\hline $593.660 \ldots \ldots \ldots \ldots$ & 13.40 & 12.61 & 11.00 & 9.93 & 9.04 & EB \\
\hline $595.795 \ldots \ldots \ldots \ldots$ & 13.47 & 12.64 & 10.97 & 9.92 & 9.02 & EB \\
\hline $596.723 \ldots \ldots \ldots \ldots$ & 13.49 & 12.60 & 10.96 & 9.91 & 9.04 & EB \\
\hline $600.822 \ldots \ldots \ldots \ldots$ & & 12.57 & 10.96 & 9.91 & 8.98 & EB \\
\hline $601.608 \ldots \ldots \ldots \ldots$ & 13.51 & 12.62 & 10.96 & 9.90 & 8.98 & EB \\
\hline $601.640 \ldots \ldots \ldots \ldots$ & & & 10.94 & & & WL \\
\hline $604.577 \ldots \ldots \ldots . . .$. & & & 10.93 & & & WL \\
\hline $605.771 \ldots \ldots \ldots \ldots$ & 13.53 & 12.61 & 10.96 & 9.87 & 8.97 & EB \\
\hline $605.644 \ldots \ldots \ldots \ldots$ & & & 10.93 & & & WL \\
\hline $606.857 \ldots \ldots \ldots \ldots$ & 13.41 & 12.58 & 10.95 & 9.86 & 8.94 & EB \\
\hline $607.670 \ldots \ldots \ldots \ldots$ & 13.43 & 12.57 & 10.94 & 9.84 & 8.94 & EB \\
\hline $608.824 \ldots \ldots \ldots \ldots$ & 13.48 & 12.57 & 10.97 & 9.86 & 8.94 & EB \\
\hline $613.763 \ldots \ldots \ldots \ldots$ & 13.55 & 12.58 & 10.98 & 9.89 & 8.95 & EB \\
\hline $615.630 \ldots \ldots \ldots \ldots$ & 13.44 & 12.61 & 10.98 & 9.88 & 8.95 & EB \\
\hline $615.656 \ldots \ldots \ldots \ldots$ & & & 10.97 & & & WL \\
\hline $616.840 \ldots \ldots \ldots \ldots$ & 13.44 & 12.61 & 10.97 & 9.86 & 8.94 & EB \\
\hline $617.614 \ldots \ldots \ldots \ldots$ & & & 10.93 & & & WL \\
\hline $618.712 \ldots \ldots \ldots \ldots$ & 13.46 & 12.60 & 10.97 & 9.88 & 8.94 & EB \\
\hline $621.648 \ldots \ldots \ldots \ldots$ & & & 10.89 & & & WL \\
\hline $623.748 \ldots \ldots \ldots \ldots$ & & & 10.96 & & & WL \\
\hline $624.506 \ldots \ldots \ldots \ldots$ & & & 10.96 & & & WL \\
\hline $625.496 \ldots \ldots \ldots \ldots$ & & & 10.93 & & & WL \\
\hline $626.501 \ldots \ldots \ldots \ldots$ & & & 10.93 & & & WL \\
\hline $627.486 \ldots \ldots \ldots \ldots$ & & & 10.88 & & & WL \\
\hline $628.510 \ldots \ldots \ldots \ldots$ & & & 10.93 & & & WL \\
\hline $629.539 \ldots \ldots \ldots \ldots$ & & & 10.96 & & & WL \\
\hline $630.503 \ldots \ldots \ldots \ldots$ & & & 10.93 & & & WL \\
\hline $632.515 \ldots \ldots \ldots \ldots$ & & & 10.89 & & & WL \\
\hline $633.546 \ldots \ldots \ldots \ldots$ & & & 10.91 & & & WL \\
\hline $634.499 \ldots \ldots \ldots \ldots$ & & & 10.88 & & & WL \\
\hline $640.569 \ldots \ldots \ldots \ldots$ & & & 10.97 & & & WL \\
\hline $642.503 \ldots \ldots \ldots \ldots$ & & & 10.91 & & & WL \\
\hline $643.482 \ldots \ldots \ldots \ldots$ & & & 10.92 & & & WL \\
\hline $644.482 \ldots \ldots \ldots \ldots$ & & & 10.91 & & & WL \\
\hline $645.478 \ldots \ldots \ldots \ldots$ & & & 10.92 & & & WL \\
\hline $646.474 \ldots \ldots \ldots \ldots$ & & & 10.96 & & & WL \\
\hline $649.478 \ldots \ldots \ldots \ldots$ & & & 10.94 & & & WL \\
\hline $651.516 \ldots \ldots \ldots \ldots$ & & & 10.86 & & & WL \\
\hline $652.500 \ldots \ldots \ldots \ldots$ & & & 10.86 & & & WL \\
\hline $653.497 \ldots \ldots \ldots \ldots$ & & & 10.89 & & & WL \\
\hline $655.512 \ldots \ldots \ldots \ldots$ & & & 10.87 & & & WL \\
\hline $661.476 \ldots \ldots \ldots \ldots$ & & & 10.85 & & & WL \\
\hline
\end{tabular}


TABLE 1-Continued

\begin{tabular}{|c|c|c|c|c|c|c|}
\hline HJD $(2,450,000+)$ & $U$ & $B$ & $V$ & $R_{\mathrm{C}}$ & $i_{\mathrm{G}}$ & Observer \\
\hline $662.484 \ldots$. & & & 10.77 & & & WL \\
\hline $683.548 \ldots \ldots \ldots \ldots$ & 13.38 & 12.50 & 10.85 & 9.74 & 8.77 & JK \\
\hline $684.542 \ldots \ldots \ldots \ldots$ & 13.45 & 12.49 & 10.86 & 9.74 & 8.79 & JK \\
\hline $685.506 \ldots \ldots \ldots \ldots$ & & & 10.86 & & 8.80 & JK \\
\hline $686.502 \ldots \ldots \ldots \ldots$ & & & 10.84 & & 8.80 & JK \\
\hline $687.520 \ldots \ldots \ldots \ldots$ & & & 10.86 & & 8.82 & JK \\
\hline $687.597 \ldots \ldots \ldots \ldots$ & & & 10.87 & & 8.82 & JK \\
\hline $689.496 \ldots \ldots \ldots \ldots$ & & & 10.87 & & 8.82 & JK \\
\hline $690.509 \ldots \ldots \ldots \ldots$ & & & 10.88 & & 8.82 & JK \\
\hline $692.468 \ldots \ldots \ldots \ldots$ & & & 10.92 & & 8.83 & JK \\
\hline $694.496 \ldots \ldots \ldots \ldots$ & 13.50 & 12.58 & 10.92 & 9.80 & 8.87 & JK \\
\hline $696.506 \ldots \ldots \ldots \ldots$ & & & 10.94 & & 8.86 & JK \\
\hline $697.493 \ldots \ldots \ldots \ldots$ & & & 10.95 & & 8.86 & JK \\
\hline $698.495 \ldots \ldots \ldots \ldots$ & & & 10.97 & & 8.87 & JK \\
\hline $699.490 \ldots \ldots \ldots \ldots$ & & & 10.96 & & 8.87 & JK \\
\hline $700.487 \ldots \ldots \ldots \ldots$ & & & 10.96 & & 8.86 & $\mathrm{JK}$ \\
\hline $701.488 \ldots \ldots \ldots \ldots$ & & & 10.95 & & 8.86 & JK \\
\hline $703.490 \ldots \ldots \ldots \ldots$ & & & 10.94 & & 8.85 & JK \\
\hline $704.491 \ldots \ldots \ldots \ldots$ & & & 10.94 & & 8.86 & JK \\
\hline $705.508 \ldots \ldots \ldots \ldots$ & 13.40 & 12.60 & 10.94 & 9.83 & 8.86 & JK \\
\hline $706.502 \ldots \ldots \ldots \ldots$ & & & 10.93 & & 8.86 & JK \\
\hline $707.498 \ldots \ldots \ldots \ldots$ & 13.44 & 12.60 & 10.93 & 9.81 & 8.86 & JK \\
\hline $712.468 \ldots \ldots \ldots \ldots$ & & 12.56 & 10.88 & & & $\mathrm{CS}$ \\
\hline $713.463 \ldots \ldots \ldots \ldots$ & & 12.54 & 10.85 & & & $\mathrm{CS}$ \\
\hline $714.471 \ldots \ldots \ldots \ldots$ & & 12.53 & 10.85 & & & $\mathrm{CS}$ \\
\hline $722.535 \ldots \ldots \ldots \ldots$ & & & 10.79 & & & WL \\
\hline $725.486 \ldots \ldots \ldots \ldots$ & & & 10.86 & & & WL \\
\hline $727.522 \ldots \ldots \ldots \ldots$ & & & 10.80 & & & WL \\
\hline $728.494 \ldots \ldots \ldots \ldots$ & & & 10.80 & & & WL \\
\hline $737.477 \ldots \ldots \ldots \ldots$ & & 12.56 & 10.87 & & & $\mathrm{CS}$ \\
\hline $741.485 \ldots \ldots \ldots \ldots$ & & 12.58 & 10.90 & & & $\mathrm{CS}$ \\
\hline $743.500 \ldots \ldots \ldots \ldots$ & & & 10.86 & & & WL \\
\hline $743.506 \ldots \ldots \ldots \ldots$ & & & 10.92 & & & $\mathrm{CS}$ \\
\hline $745.517 \ldots \ldots \ldots \ldots$ & & & 10.92 & & & WL \\
\hline $746.496 \ldots \ldots \ldots \ldots$ & & & 10.93 & & & WL \\
\hline $750.511 \ldots \ldots \ldots \ldots$ & & & 10.90 & & & WL \\
\hline $752.499 \ldots \ldots \ldots \ldots$ & & & 10.90 & & & WL \\
\hline $761.513 \ldots \ldots \ldots \ldots$ & & & 10.88 & & & WL \\
\hline $765.519 \ldots \ldots \ldots \ldots$ & & & 10.84 & & & WL \\
\hline $769.520 \ldots \ldots \ldots \ldots$ & & & 10.75 & & & WL \\
\hline $773.522 \ldots \ldots \ldots \ldots$ & & & 10.76 & & & WL \\
\hline $853.850 \ldots \ldots \ldots \ldots$ & & & 11.88 & & & WL \\
\hline $853.886 \ldots \ldots \ldots \ldots$ & & & 12.20 & & & WL \\
\hline $854.853 \ldots \ldots \ldots \ldots$ & & & 12.15 & & & WL \\
\hline $856.885 \ldots \ldots \ldots \ldots$ & & & 12.22 & & 9.78 & MJ \\
\hline $857.900 \ldots \ldots \ldots \ldots$ & 15.21 & 14.17 & 12.27 & 10.97 & 9.83 & MJ \\
\hline $858.842 \ldots \ldots \ldots \ldots$ & 14.96 & 14.17 & 12.30 & 10.98 & 9.84 & MJ \\
\hline $859.882 \ldots \ldots \ldots \ldots$ & 15.04 & 14.24 & 12.34 & 11.03 & 9.87 & MJ \\
\hline $860.877 \ldots \ldots \ldots$ & 15.20 & 14.27 & 12.36 & 11.04 & 9.88 & MJ \\
\hline $862.858 \ldots \ldots \ldots \ldots$ & 15.10 & 14.26 & 12.34 & 11.05 & 9.90 & MJ \\
\hline 865.899 ............ & & 14.32 & 12.36 & 11.05 & 9.85 & MJ \\
\hline $866.864 \ldots \ldots \ldots \ldots$ & & 99.00 & 12.34 & 11.05 & 9.86 & MJ \\
\hline $868.882 \ldots \ldots \ldots \ldots$ & 15.31 & 14.31 & 12.36 & 11.04 & 9.89 & MJ \\
\hline $869.894 \ldots \ldots \ldots \ldots$ & 15.22 & 14.35 & 12.37 & 11.06 & 9.89 & MJ \\
\hline $870.399 \ldots \ldots \ldots \ldots$ & 15.24 & 14.37 & 12.40 & 11.07 & 9.89 & MJ \\
\hline $871.894 \ldots \ldots \ldots \ldots$ & 15.16 & 14.46 & 12.40 & 11.08 & 9.90 & MJ \\
\hline $872.393 \ldots \ldots \ldots \ldots$ & 15.16 & 14.40 & 12.41 & 11.09 & 9.91 & MJ \\
\hline $873.893 \ldots \ldots \ldots \ldots$ & 15.37 & 14.41 & 12.41 & 11.10 & 9.92 & MJ \\
\hline $874.897 \ldots \ldots \ldots \ldots$ & 15.28 & 14.45 & 12.43 & 11.11 & 9.91 & MJ \\
\hline $875.841 \ldots \ldots \ldots \ldots$ & & & 12.40 & & & WL \\
\hline $875.899 \ldots \ldots \ldots \ldots$ & 15.36 & 14.47 & 12.43 & 11.11 & 9.92 & MJ \\
\hline $876.901 \ldots \ldots \ldots \ldots$ & 15.48 & 14.48 & 12.46 & 11.11 & 9.92 & MJ \\
\hline 877.909 ............ & 15.42 & 14.46 & 12.46 & 11.10 & 9.92 & MJ \\
\hline $878.906 \ldots \ldots \ldots \ldots$ & 15.47 & 14.48 & 12.45 & 11.11 & 9.91 & MJ \\
\hline $879.883 \ldots \ldots \ldots \ldots$ & & & 12.54 & & & WL \\
\hline $880.876 \ldots \ldots \ldots \ldots$ & & & 12.55 & & & WL \\
\hline $881.911 \ldots \ldots \ldots \ldots$ & 15.44 & 14.48 & 12.43 & 11.08 & 9.89 & MJ \\
\hline
\end{tabular}


TABLE 1-Continued

\begin{tabular}{|c|c|c|c|c|c|c|}
\hline HJD $(2,450,000+)$ & $U$ & $B$ & $V$ & $R_{\mathrm{C}}$ & $i_{\mathrm{G}}$ & Observer \\
\hline $887.847 \ldots \ldots \ldots \ldots$ & & 14.33 & 12.30 & & & CS \\
\hline $888.843 \ldots \ldots \ldots \ldots$ & & 14.31 & 12.29 & & & $\mathrm{CS}$ \\
\hline $890.825 \ldots \ldots \ldots \ldots$ & & & 12.33 & & & WL \\
\hline $890.869 \ldots \ldots \ldots \ldots$ & & 14.28 & 12.17 & & & CS \\
\hline $891.888 \ldots \ldots \ldots \ldots$ & & & 12.34 & & & WL \\
\hline $891.896 \ldots \ldots \ldots \ldots$ & & 14.26 & 12.23 & & & $\mathrm{CS}$ \\
\hline $892.811 \ldots \ldots \ldots \ldots$ & & 14.25 & 12.22 & & & $\mathrm{CS}$ \\
\hline $893.915 \ldots \ldots \ldots \ldots$ & 15.52 & 14.28 & 12.22 & 10.86 & 9.71 & HD \\
\hline $894.850 \ldots \ldots \ldots \ldots$ & & & 12.34 & & & WL \\
\hline $894.921 \ldots \ldots \ldots \ldots$ & 15.38 & 14.22 & 12.20 & 10.85 & 9.68 & HD \\
\hline $895.885 \ldots \ldots \ldots \ldots$ & 15.31 & 14.23 & 12.20 & 10.85 & 9.68 & HD \\
\hline $896.809 \ldots \ldots \ldots \ldots$ & & & 12.29 & & & WL \\
\hline $897.816 \ldots \ldots \ldots \ldots$ & & & 12.36 & & & WL \\
\hline $899.837 \ldots \ldots \ldots \ldots$ & & & 12.20 & & & WL \\
\hline $901.818 \ldots \ldots \ldots \ldots$ & & & 12.22 & & & WL \\
\hline $909.872 \ldots \ldots \ldots \ldots$ & & & 12.22 & & & WL \\
\hline $910.788 \ldots \ldots \ldots \ldots$ & & & 12.25 & & & WL \\
\hline $918.881 \ldots \ldots \ldots \ldots$ & & 14.02 & 12.01 & & & TA \\
\hline $919.871 \ldots \ldots \ldots \ldots$ & & & 12.11 & & & WL \\
\hline $921.753 \ldots \ldots \ldots \ldots$ & & & 12.19 & & & WL \\
\hline $922.793 \ldots \ldots \ldots \ldots$ & & & 11.97 & & & TA \\
\hline $923.693 \ldots \ldots \ldots \ldots$ & & & 12.14 & & & WL \\
\hline $925.670 \ldots \ldots \ldots \ldots$ & & & 12.19 & & & WL \\
\hline $926.782 \ldots \ldots \ldots \ldots$ & & & 12.11 & & & WL \\
\hline $927.701 \ldots \ldots \ldots \ldots$ & & & 12.02 & & & WL \\
\hline $931.770 \ldots \ldots \ldots \ldots$ & & & 11.93 & & & WL \\
\hline $934.764 \ldots \ldots \ldots \ldots$ & & & 11.84 & & & WL \\
\hline $937.716 \ldots \ldots \ldots \ldots$ & & & 11.79 & & & WL \\
\hline $941.741 \ldots \ldots \ldots \ldots$ & & & 11.84 & & & WL \\
\hline $949.801 \ldots \ldots \ldots \ldots$ & & & 11.88 & & & WL \\
\hline $950.723 \ldots \ldots \ldots \ldots$ & & & 11.65 & & 9.28 & PW \\
\hline $963.625 \ldots \ldots \ldots \ldots$ & & & 12.20 & & & WL \\
\hline $965.711 \ldots \ldots \ldots \ldots$ & & & 12.39 & & & WL \\
\hline $971.701 \ldots \ldots \ldots \ldots$ & & & 12.36 & & & WL \\
\hline $980.628 \ldots \ldots \ldots \ldots$ & & & 12.07 & & & WL \\
\hline $994.498 \ldots \ldots \ldots \ldots$ & & & 11.86 & & & WL \\
\hline $996.647 \ldots \ldots \ldots \ldots$ & & & 11.77 & & & WL \\
\hline $997.508 \ldots \ldots \ldots \ldots$ & & & 11.78 & & & WL \\
\hline $1000.505 \ldots \ldots \ldots \ldots$ & & & 11.71 & & & WL \\
\hline $1004.522 \ldots \ldots \ldots \ldots$ & & & 11.66 & & & WL \\
\hline $1007.495 \ldots \ldots \ldots \ldots$ & & & 11.65 & & & WL \\
\hline $1011.517 \ldots \ldots \ldots \ldots$ & & & 11.65 & & & WL \\
\hline $1012.478 \ldots \ldots \ldots \ldots$ & & & 11.66 & & & WL \\
\hline $1013.542 \ldots \ldots \ldots \ldots$ & & & 11.71 & & & WL \\
\hline $1014.466 \ldots \ldots \ldots \ldots$ & & & 11.67 & & & WL \\
\hline $1016.520 \ldots \ldots \ldots$ & & & 11.72 & & & WL \\
\hline $1019.506 \ldots \ldots \ldots \ldots$ & & & 11.84 & & & WL \\
\hline $1020.491 \ldots \ldots \ldots \ldots$ & & & 11.77 & & & WL \\
\hline $1023.484 \ldots \ldots \ldots \ldots$ & & & 12.02 & & & WL \\
\hline $1026.517 \ldots \ldots \ldots .$. & & & 12.06 & & & WL \\
\hline $1029.486 \ldots \ldots \ldots \ldots$ & & & 12.27 & & & WL \\
\hline $1031.489 \ldots \ldots \ldots$. & & & 12.12 & & & WL \\
\hline $1034.510 \ldots \ldots \ldots .$. & & & 12.36 & & & WL \\
\hline $1034.717 \ldots \ldots \ldots$ & 15.58 & 14.26 & 12.29 & 10.95 & 9.80 & JA \\
\hline $1035.531 \ldots \ldots \ldots$. & & & 12.33 & & 9.80 & JA \\
\hline $1036.481 \ldots \ldots \ldots \ldots$ & & & 12.48 & & & WL \\
\hline $1036.773 \ldots \ldots \ldots . .$. & & & 12.38 & & 9.86 & $\mathrm{JA}$ \\
\hline $1037.520 \ldots \ldots \ldots \ldots$ & & & 12.30 & & & WL \\
\hline $1037.640 \ldots \ldots \ldots \ldots$ & & & 12.47 & & 9.94 & JA \\
\hline $1038.527 \ldots \ldots \ldots \ldots$ & & & 12.55 & & & WL \\
\hline $1038.664 \ldots \ldots \ldots \ldots$ & & & 12.54 & & 9.99 & JA \\
\hline $1039.492 \ldots \ldots \ldots \ldots$ & & & 12.68 & & & WL \\
\hline $1039.637 \ldots \ldots \ldots \ldots$ & & & 12.61 & & 10.04 & $\mathrm{JA}$ \\
\hline $1040.752 \ldots \ldots \ldots \ldots$ & & & 12.63 & & 10.07 & JA \\
\hline $1041.610 \ldots \ldots \ldots . .$. & 16.26 & 14.81 & 12.72 & 11.34 & 10.15 & JA \\
\hline $1042.700 \ldots \ldots \ldots$ & & & 12.76 & & 10.15 & JA \\
\hline $1043.519 \ldots \ldots \ldots$. & & & 12.76 & & & WL \\
\hline
\end{tabular}


TABLE 1-Continued

\begin{tabular}{|c|c|c|c|c|c|c|}
\hline $\operatorname{HJD}(2,450,000+)$ & $U$ & $B$ & $V$ & $R_{\mathrm{C}}$ & $i_{\mathrm{G}}$ & Observer \\
\hline $1043.549 \ldots \ldots \ldots$ & & & 12.80 & & 10.19 & JA \\
\hline $1044.696 \ldots \ldots \ldots \ldots$ & & & 12.84 & & 10.23 & JA \\
\hline $1045.677 \ldots \ldots \ldots \ldots$ & & & 12.88 & & 10.26 & JA \\
\hline $1046.666 \ldots \ldots \ldots \ldots$ & & & 12.92 & & 10.28 & JA \\
\hline $1047.647 \ldots \ldots \ldots \ldots$ & & & 12.94 & & 10.30 & JA \\
\hline $1048.664 \ldots \ldots \ldots \ldots$ & 16.74 & 15.11 & 12.98 & 11.54 & 10.30 & JA \\
\hline $1049.549 \ldots \ldots \ldots$ & & & 12.98 & & 10.32 & JA \\
\hline $1051.624 \ldots \ldots \ldots \ldots$ & 16.82 & 15.18 & 13.01 & 11.56 & 10.35 & JA \\
\hline $1052.724 \ldots \ldots \ldots \ldots$ & & & 12.98 & & 10.36 & JA \\
\hline $1053.481 \ldots \ldots \ldots$. & & & 13.04 & & 10.38 & JA \\
\hline $1054.541 \ldots \ldots \ldots \ldots$ & & & 13.07 & & 10.38 & JA \\
\hline $1055.549 \ldots \ldots \ldots$ & 16.84 & 15.22 & 13.07 & 11.64 & 10.40 & JA \\
\hline $1059.668 \ldots \ldots \ldots \ldots$ & & 15.28 & 13.14 & 11.68 & 10.46 & JA \\
\hline $1061.659 \ldots \ldots \ldots$ & & & 13.20 & & 10.55 & JA \\
\hline $1062.661 \ldots \ldots \ldots \ldots$ & 17.01 & 15.34 & 13.27 & 11.82 & 10.59 & JA \\
\hline $1063.576 \ldots \ldots \ldots \ldots$ & 17.11 & 15.53 & 13.32 & 11.88 & 10.63 & $\mathrm{CS}$ \\
\hline $1064.579 \ldots \ldots \ldots$ & & 15.50 & 13.39 & & & $\mathrm{CS}$ \\
\hline $1065.578 \ldots \ldots \ldots \ldots$ & 17.22 & 15.55 & 13.43 & 11.97 & 10.74 & $\mathrm{CS}$ \\
\hline $1067.593 \ldots \ldots \ldots$ & 17.40 & 15.74 & 13.64 & 12.16 & 10.91 & $\mathrm{CS}$ \\
\hline $1068.626 \ldots \ldots \ldots \ldots$ & 17.56 & 15.85 & 13.72 & 12.25 & 10.98 & $\mathrm{CS}$ \\
\hline $1069.619 \ldots \ldots \ldots \ldots$ & 17.66 & 15.99 & 13.85 & 12.38 & 11.08 & $\mathrm{CS}$ \\
\hline $1070.554 \ldots \ldots \ldots$ & 17.83 & 16.11 & 13.96 & 12.47 & 11.19 & CS \\
\hline $1071.501 \ldots \ldots \ldots$ & & & 14.03 & & & WL \\
\hline $1071.625 \ldots \ldots \ldots \ldots$ & 17.98 & 16.24 & 14.10 & 12.55 & 11.29 & $\mathrm{CS}$ \\
\hline $1072.617 \ldots \ldots \ldots \ldots$ & 18.28 & 16.45 & 14.26 & 12.73 & 11.42 & $\mathrm{CS}$ \\
\hline $1074.475 \ldots \ldots \ldots \ldots$ & & & 14.23 & & & WL \\
\hline $1075.506 \ldots \ldots \ldots \ldots$ & & & {$[15$.} & & & WL \\
\hline $1078.515 \ldots \ldots \ldots \ldots$ & & & {$[15$.} & & & WL \\
\hline $1081.532 \ldots \ldots \ldots \ldots$ & & & {$[15$.} & & & WL \\
\hline $1094.529 \ldots \ldots \ldots \ldots$ & & & {$[15$.} & & & WL \\
\hline $1097.521 \ldots \ldots \ldots \ldots$ & & & {$[15$.} & & & WL \\
\hline $1101.509 \ldots \ldots \ldots \ldots$ & & & {$[15$.} & & & WL \\
\hline $1110.509 \ldots \ldots \ldots$ & & & {$[15$.} & & & WL \\
\hline $1115.529 \ldots \ldots \ldots \ldots$ & & & {$[15$.} & & & WL \\
\hline $1125.516 \ldots \ldots \ldots \ldots$ & 19.75 & 17.62 & 15.14 & 13.42 & 11.94 & $\mathrm{AM}$ \\
\hline $1126.524 \ldots \ldots \ldots \ldots$ & 19.82 & 17.60 & 15.14 & 13.43 & 11.93 & $\mathrm{AM}$ \\
\hline $1127.507 \ldots \ldots \ldots \ldots$ & & 17.69 & 15.17 & 13.44 & 11.97 & $\mathrm{AM}$ \\
\hline $1128.514 \ldots \ldots \ldots \ldots$ & & & 15.20 & & 11.98 & $\mathrm{AM}$ \\
\hline $1129.507 \ldots \ldots \ldots \ldots$ & & 17.69 & 15.21 & 13.45 & 11.98 & $\mathrm{AM}$ \\
\hline $1130.503 \ldots \ldots \ldots \ldots$ & & & 15.22 & & 12.00 & $\mathrm{AM}$ \\
\hline $1130.628 \ldots \ldots \ldots \ldots$ & & & 15.13 & & & WL \\
\hline $1234.87 \ldots \ldots \ldots \ldots$ & & 19.187 & 16.45 & 14.54 & 12.82 & $\mathrm{RD}$ \\
\hline $1235.86 \ldots \ldots \ldots \ldots$ & & & 16.61 & & 12.93 & $\mathrm{RD}$ \\
\hline $1236.89 \ldots \ldots \ldots \ldots$ & & & 16.81 & & 13.06 & $\mathrm{RD}$ \\
\hline $1238.87 \ldots \ldots \ldots \ldots$ & & 20.31 & 17.26 & 15.15 & 13.34 & RD \\
\hline $1260.837 \ldots \ldots \ldots \ldots$ & & & 20.10 & & & $\mathrm{CS}$ \\
\hline $1261.871 \ldots \ldots \ldots \ldots$ & & & 19.90 & & & $\mathrm{CS}$ \\
\hline $1262.857 \ldots \ldots \ldots \ldots$ & & & 19.67 & & & $\mathrm{CS}$ \\
\hline $1263.866 \ldots \ldots \ldots \ldots$ & & & 20.15 & & & $\mathrm{CS}$ \\
\hline $1264.876 \ldots \ldots \ldots \ldots$ & & & 19.92 & & & $\mathrm{CS}$ \\
\hline $1265.910 \ldots \ldots \ldots \ldots$ & & & 19.84 & & & $\mathrm{CS}$ \\
\hline $1267.865 \ldots \ldots \ldots \ldots$ & & & 20.08 & 17.72 & 15.56 & $\mathrm{CS}$ \\
\hline $1368.06 \ldots \ldots \ldots \ldots$ & 23.48 & & 22.10 & 20.87 & 18.51 & $\mathrm{TN}$ \\
\hline
\end{tabular}

NotE--Observer (at $0.91 \mathrm{~m}$ Dutch telescope, unless stated otherwise): JA: J. Arts, TA: T. Arentoft, SB: S. Benetti, TN: S. Benetti, $3.5 \mathrm{~m}$ TNG at La Palma, EB: E. Brogt, RD: R. Dijkstra, HD: H.W. Duerbeck, MJ: M. Janson, JK: J. Kurk, WL: W. Liller (0.2 m telescope, Reñaca Bajo), AM: A. van der Meer, CS: C. Sterken, MT: M. Turatto, TV: T. Voskes, PW: P. de Wildt.

The UBVRiz observations made in 1996 by the ChileanEuropean group were published as Duerbeck et al. (1997, hereafter D97). In the present paper, photometric observations for the years 1997, 1998, and 1999 are reported, covering the complete rise and decline of the brightness of V4334 Sgr in the optical region (§ 2). Its properties before the outburst are investigated, and constraints are given on its distance $(\S 3)$. The light and color curves are constructed and interpreted $(\S 4)$. The observed fadings are explained by dust formation, and similarities and differences of these events with fadings of R CrB stars are outlined $(\S 5)$. The development of the energy distribution between 0.36 and 15 $\mu \mathrm{m}$ is investigated, and parallels to dust-forming classical novae are shown (§ 6). Finally, the timescales of the final $\mathrm{He}$ 
flashes in V4334 Sgr, V605 Aql, and FG Sge are compared, and predictions for their future evolution are given $(\S 7)$.

\section{OBSERVATIONS}

$U B V R i$ observations of V4334 Sgr were carried out with the $0.91 \mathrm{~m}$ Dutch light collector at ESO La Silla until its shutdown on 1999 April 1. Filters and comparison stars were the same as those used in D97 (the filters $R$ and $i$ refer to Cousins $R_{\mathrm{C}}$ and Gunn $i_{\mathrm{G}}$, respectively). The object was also observed until 1998 October with the $0.2 \mathrm{~m} \mathrm{f} / 1.5$ Schmidt telescope of W. Liller in Reñaca, Viña del Mar, Chile. Additional observations were obtained with the $3.5 \mathrm{~m}$ Telescopio Nazionale Galileo (TNG) ${ }^{6}$ in 1999 July.

The early observations with the Dutch telescope were analyzed using ROMAFOT aperture photometry. The continuing decline in brightness, especially at short wavelengths, made it necessary to carry out DAOPHOT profile-fitting photometry of V4334 Sgr from 1997 September 15 onward. Because of the increase of exposure times, comparison star 1 of D97 became too bright to be used as a local standard, and the average magnitude of stars 2-6 (taken from D97) was used as the reference magnitude in all filters. The Reñaca observations are based on CCD aperture photometry. Since they were always taken relative to star 1 through a nonstandard $V$ filter, which extends toward the red, a transformation was established from simultaneous Dutch $(V, i)$ and Reñaca $\left(\Delta V^{*}\right)$ observations, which makes it possible to convert the Reñaca observations into the standard $V$ system

$$
V=10.65+0.93 \Delta V^{*}+0.047(V-i),
$$

where $\Delta V^{*}$ is the magnitude difference relative to the bright comparison star; the color $V-i$ is taken from the Dutch observations taken near the time of the Reñaca observations. The $U B V R i$ data of 1997-1999 are listed in Table 1.

\section{V4334 SAGITTARII BEFORE ITS FINAL He FLASH}

V4334 Sgr, before its final He flash, was a faint blue star on the ESO/SERC Sky Atlas (Duerbeck \& Benetti 1996, hereafter D96). In this section, the apparent magnitude is determined, the interstellar reddening is estimated, and

${ }^{6}$ TNG is operated on the island of La Palma by the Centro Galileo Galilei of the Consorzio Nazionale per l'Astronomia e l'Astrofisica at the Spanish Observatorio del Roque de los Muchachos of the Instituto de Astrofísica de Canarias. upper and lower limits of its distance are given. This information is important for the derivation of the luminosity during the final He flash phase.

Deep images with the TNG telescope in 1999 July allow us to establish a preliminary faint magnitude scale in the vicinity of V4334 Sgr (Fig. 1). A northern and a southern visual companion, each 2".5 from V4334 Sgr, are seen on the Sky Atlas images (see Plate 1 of D96). The northern companion has $V=20.85, R=19.93$, and the southern one $V=21.12, R=20.30$. Because of the lack of $B$ magnitudes in the TNG observations, pseudo $B$ magnitudes were assigned to some stars with the help of the available $U$ and $V$ magnitudes. Using this preliminary scale, the visibility of some fainter field stars was checked on the Sky Atlas plates, and the preoutburst photographic magnitudes of V4334 Sgr were estimated to be $m_{B} \approx 21 \mathrm{mag}, m_{R}>21.5 \mathrm{mag}$.

The interstellar reddening of V4334 Sgr is still poorly known. While $E_{B-V}=0.54$ was estimated by D 96 , the value $0.71 \pm 0.09$ was derived by Pollacco (1999) from the observed $\mathrm{H} \alpha / \mathrm{H} \beta$ line ratio of the surrounding planetary nebula for case B. Another value, 1.15, was suggested by Eyres et al. (1998b). Kimeswenger \& Kerber (1998) made a detailed study of interstellar reddening in the field around V4334 Sgr and found $E_{B-V}=0.90 \pm 0.09$ for 18 stars with distances $d \geq 2 \mathrm{kpc}$. Since we have reasons to believe that V4334 Sgr has a distance $d \geq 2 \mathrm{kpc}$ (see $\S 6$ ), we adopted $E_{B-V}=0.8$, which is compatible with both Pollacco's and Kimeswenger \& Kerber's results.

The brightness of the preoutburst magnitude of V4334 Sgr was compared with other PNNs. Absolute $B$ magnitudes of central stars of planetary nebulae were derived using information on trigonometric parallaxes given in Jacoby, De Marco, \& Sawyer (1998) and Acker et al. (1998). Apparent magnitudes were taken from the catalogue of Acker et al. (1992), the interstellar extinction was calculated with the model of Hakkila et al. (1997). Our sample is restricted to central stars in roundish bright and faint nebulae, while central stars inside irregular nebulae and stars that are obviously blended with field stars were not considered. The derived absolute magnitudes are listed in Table 2. Using the average value and its $1 \sigma$ deviation, $M_{B}=6.3 \pm 1.2$, and taking $m_{B}=21 \mathrm{mag}$ for the preoutburst magnitude of V4334 Sgr, its distance is derived to $1900_{-800}^{+1400} \mathrm{pc}$, assuming a reddening $E_{B-V}=0.8$. The value $E_{B-V}=0.7$ would increase the distance estimates by $20 \%$. Jacoby et al. (1998) concluded that the distance remains poorly determined, with possible values lying in the range 1

TABLE 2

Absolute $B$ Magnitudes of Central Stars of Selected Planetary Nebulae

\begin{tabular}{ccccccc}
\hline \hline Object & $\begin{array}{c}d \\
(\mathrm{pc})\end{array}$ & Source & \multicolumn{1}{c}{$m_{B}$} & $A_{B}$ & $M_{B}$ & Comment \\
\hline NGC $6720 \ldots \ldots$ & 704 & USNO & 15.3 & 0.32 & 5.74 & Ring nebula \\
NGC $6853 \ldots \ldots$ & 380 & USNO & 13.66 & 0.74 & 5.12 & \\
NGC $7293 \ldots \ldots$ & 213 & USNO & 13.10 & 0.08 & 6.38 & Helix nebula \\
Abell $31 \ldots \ldots \ldots$ & 211 & USNO & 15.20 & 0.07 & 8.51 & \\
Abell $36 \ldots \ldots \ldots$ & 243 & Hipp & 11.28 & 0.11 & 4.24 & \\
Abell $74 \ldots \ldots \ldots$ & 752 & USNO & 16.91 & 0.37 & 7.16 & \\
PHL $932 \ldots \ldots$. & 110 & Hipp & 11.83 & 0.09 & 6.53 & \\
PW1 $\ldots \ldots \ldots \ldots$ & 433 & USNO & 15.3 & 0.44 & 6.68 & \\
Sh $216 \ldots \ldots \ldots$. & 130 & USNO & $12.3 \mathrm{pg}$ & 0.28 & 6.45 & \\
\hline
\end{tabular}

NoTE.-USNO = parallax from US Naval Observatory; Hipp = parallax from Hipparcos catalogue (Jacoby et al. 1998; Acker et al. 1998). 


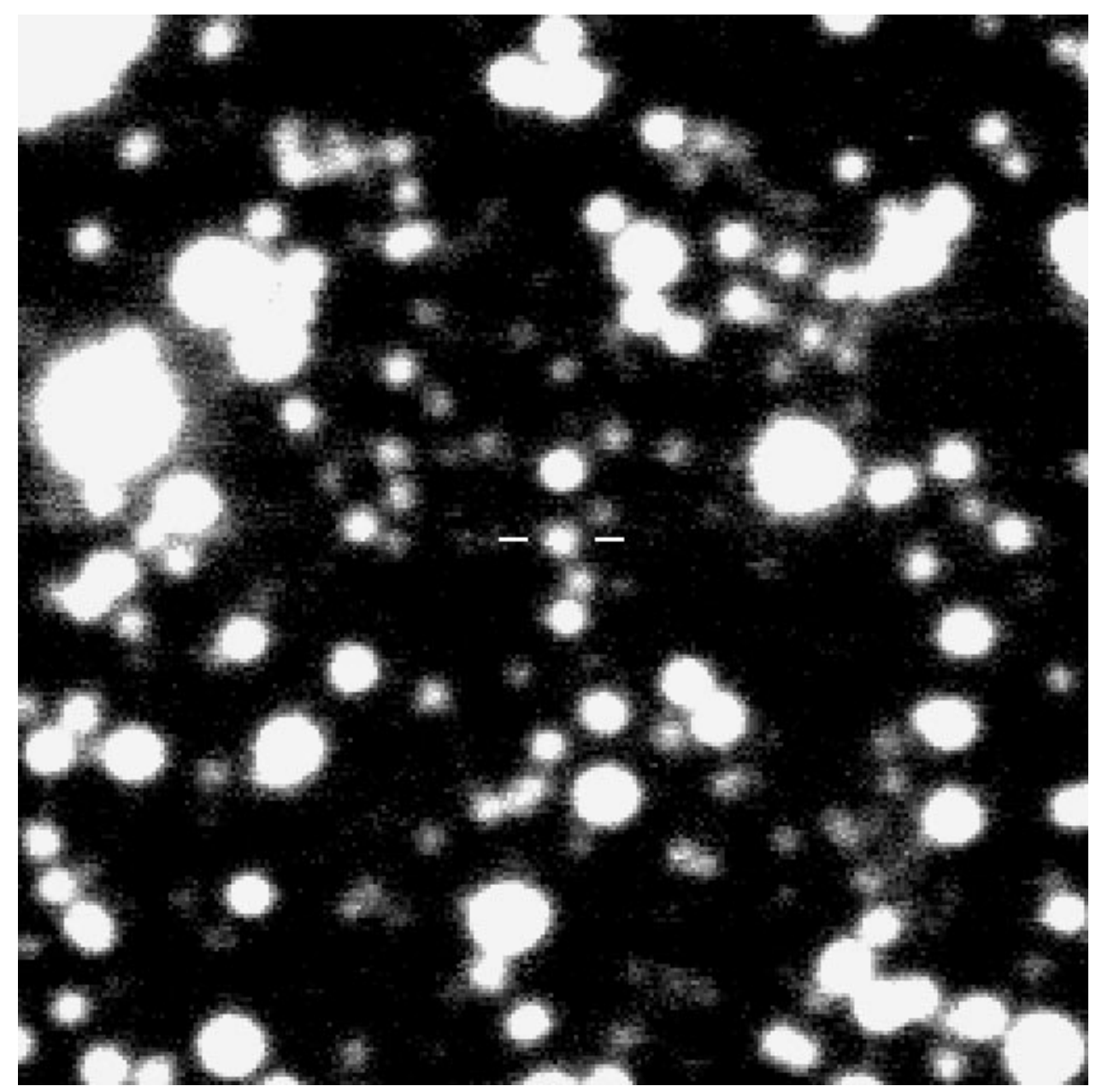

Fig. 1.-Field of V4334 Sgr, taken with the $3.5 \mathrm{~m} \mathrm{TNG}$ at La Palma through an $R$ filter in $1999 \mathrm{July}$. The object is marked. Its brightness is $R=20.3 \mathrm{mag}$. The size of the field is $40^{\prime \prime} \times 40^{\prime \prime}$. North is to the top, east to the left.

to $4 \mathrm{kpc}$. This is in agreement with the present result. This issue will be taken up again in $\S 6$.

\section{THE OPTICAL OUTBURST BEHAVIOR OF V4334 SAGITTARII}

\subsection{The Light Curve}

Figure 2 shows the complete $V$ light curve of V4334 Sgr. It can be divided into four characteristic stages, conveniently separated by the seasonal gaps when the star was too close to the Sun.

The first stage is the "rise to maximum" of 1994-1995, which is covered only by Takamizawa's 12 prediscovery observations (Takamizawa 1997; see D97 for a discussion of the prediscovery light curve). The second one is the "maximum stage" of 1996 and 1997, when quasiperiodic brightness fluctuations were superposed on an almost constant $V$ magnitude of $\approx 11$ mag. The third one is the "dust onset stage" of early and mid-1998, when the object dropped in brightness by 1 mag and continued to show quasiperiodic fluctuations. Finally, the fourth one is the "massive dust stage" of late 1998 and 1999, when the object suffered dramatic declines of 3 to $11 \mathrm{mag}$ in visible light and when strong, erratic brightness fluctuations - unfortunately poorly documented - were present.

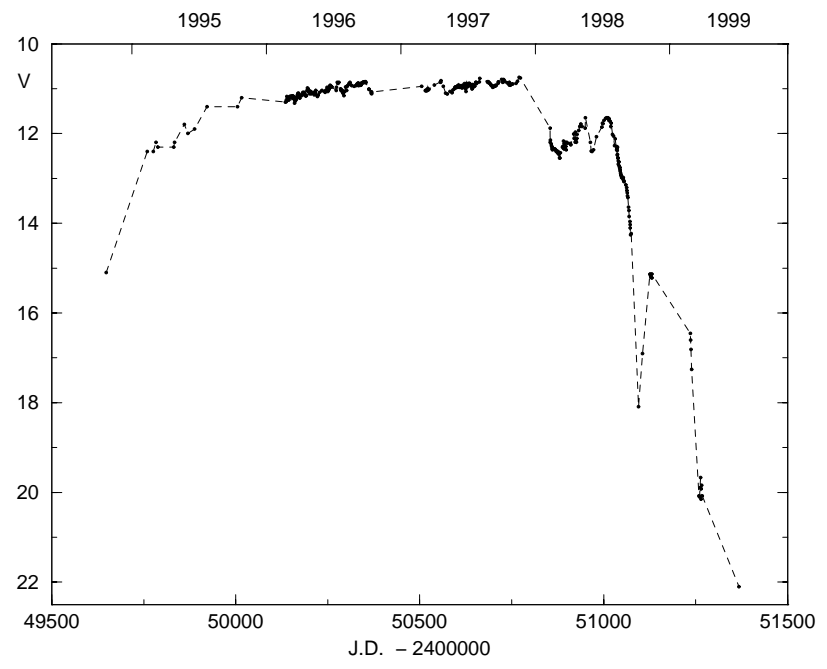

FIG. 2. - $V$ light curve of V4334 Sgr, 1995-1999. The data of 1994-1995 are the photographic prediscovery observations of Takamizawa converted to visual magnitudes, assuming that the object is expanding at constant luminosity. Note that the 1994 point is possibly only an upper limit. Some observations by Jacoby \& De Marco (1998) and Jacoby (1999) are also included. 
As already shown in D97, the first and second stages can be explained by an object with a slowly growing photosphere or pseudo photosphere, radiating almost at constant luminosity. In contrast to a stable photosphere, a pseudo photosphere is formed in a optically thick wind, which is driven by radiation pressure from an object radiating near Eddington luminosity (see, e.g., Bath \& Harkness 1989). Such a behavior is found in classical novae at early outburst stages. Dynamical instabilities causing mass loss and dust formation have also been suggested for R CrB stars, which radiate close to the Eddington limit (Asplund 1998). The photosphere of V4334 Sgr may be a true photosphere or a pseudo photosphere; an attempt to decide between the two cases will be made later $(\S 6)$. The expansion and cooling of the photosphere is documented in the $U B V R i$ light curves (Fig. 3), which show a shift of the radiation maximum toward longer wavelengths at later times.

Quasi-periodic or cyclic fluctuations are superposed on this general photometric evolution. These variations can most easily be traced in the $V$ light curve, because the temporal coverage is highest and because the effects of the temperature decline are least noticeable in $V$. Figure 4, which also includes observations of the Russian group (see $\S 1$ ), shows the $V$ fluctuations in detail. For each of the years 1996, 1997, and 1998, the linear long-term trend in brightness was removed. In 1996, oscillations of short duration (26, 22, and 16 days and some shorter ones) are superposed on a 70 day oscillation. In 1997, a clear preference of a single oscillation with an amplitude of about $0.18 \pm 0.03$ in $V$ and a period of 56 days, prevailing over more than four cycles is seen. Secondary features are less important than in the year before (see also D97 and Duerbeck et al. 1998).

The 1998 observations belong already to the third stage, which is influenced by dust. It is difficult to decide whether the fluctuations seen in early and mid-1998 are still caused by a pulsation or by dust obscuration events, especially since the multicolor coverage is poor. A characteristic timescale of $74 \pm 8$ days is seen, but the periodicity is poorly defined and masked by the strong brightness decline that occurred in the second half of 1998. The amplitude in $V$ has increased to $0.75 \mathrm{mag}$. In the fourth stage of late 1998 and of

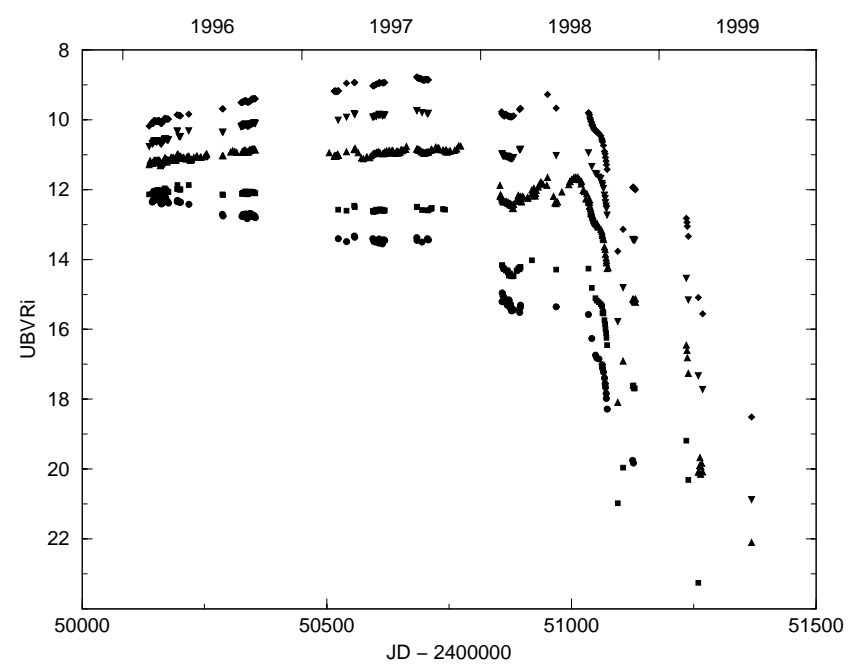

FIG. 3.- $U B V R i$ light curve of V4334 Sgr. This curve includes only the observations made at the Dutch $0.91 \mathrm{~m}$ telescope and the Renaca $0.2 \mathrm{~m}$ telescope, plus some observations at late stages made by Jacoby, Jacoby \& De Marco and Benetti. Bottom to top: $U, B, V, R, i$ light curves.

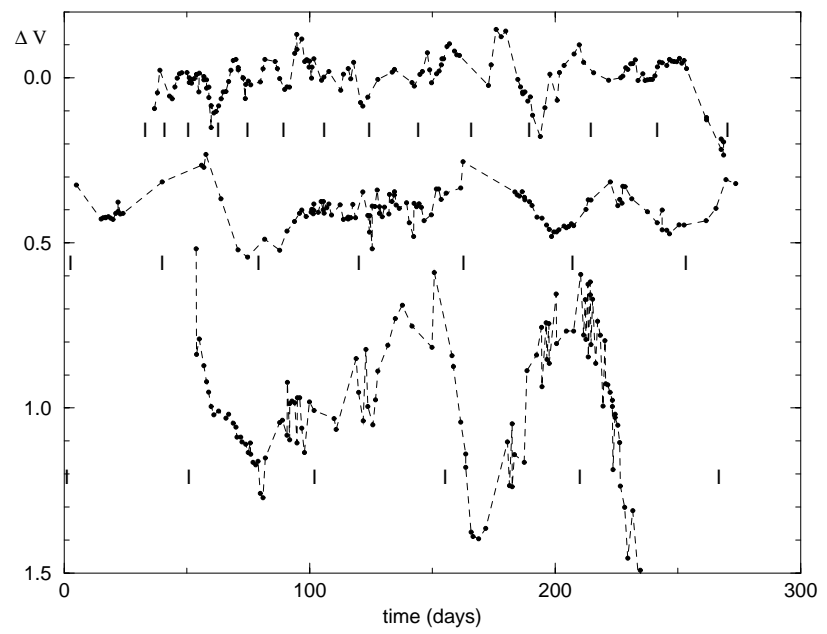

FIG. 4.-Brightness oscillations of V4334 Sgr superposed on the $V$ light curve. All available $V$ data have been used for this diagram. The long-term brightness decline of the years 1996, 1997, and 1998 (top to bottom) has been removed to show the oscillations more clearly. The data of 1997 and 1998 were shifted by 0.4 and 0.9 mag to avoid overlaps. Times of brightness minimum as described by a formula of Arkhipova et al. (1999) are marked by vertical bars placed below the corresponding light curve.

1999, the object has faded dramatically. The poor temporal coverage, as well as the strong brightness fluctuations make it impossible to study any underlying periodicities.

Summing up, the light curve between 1996 and mid-1998 can be described as being superposed by quasiperiodic fluctuations of increasing cycle length and amplitude. Arkhipova et al. (1999) claim that the variations can be described with a single period that increases linearly with time. Their ephemeris

$$
\mathrm{JD}(\min )=2,450,133.1+6.91048 E+0.871788 E^{2}
$$

was used to calculate the moments of minimum light, which are shown as vertical bars for the years 1996-1998 in Figure 4. While a trend toward longer periods and larger amplitudes at later times is clearly present, the representation of minima by the above formula is not satisfactory, and the occurrence of both well-expressed and marginal maxima and minima must be explained by the superposition of several pulsation modes, as was outlined in D87.

\subsection{V4334 Sgr in the Two-Color Diagrams}

The growth of the photosphere and the dust formation is studied in the multicolor light curve (Fig. 3) and in twocolor diagrams. The development of V4334 $\mathrm{Sgr}$ in the $(U-B$ vs. $B-V),(V-R$ vs. $B-V)$, and $(V-i$ vs. $B-V)$ two-color diagrams is shown in Figures 5 and 6 . The colors are dereddened for $E_{B-V}=0.8$. The observed averaged color indices for time intervals of days up to several weeks and, supplemented by observations of other authors at important phases, are given in Table 3.

The behavior of V4334 $\mathrm{Sgr}$ in the maximum stage is as follows. In 1996 the star was continuously cooling. In Figure 5, it is moving along the two-color track of hydrogen-deficient carbon stars of hot to intermediate temperature, as calculated by Asplund (1997, private communication). This phase was interpreted by D97 as an object radiating at almost constant luminosity, while its photosphere is slowly moving outward with a velocity of 1 $\mathrm{km} \mathrm{s}^{-1}$. In 1997, the star had moved away from the twocolor track and kept similar $U-B, B-V, V-R, V-i$ color 


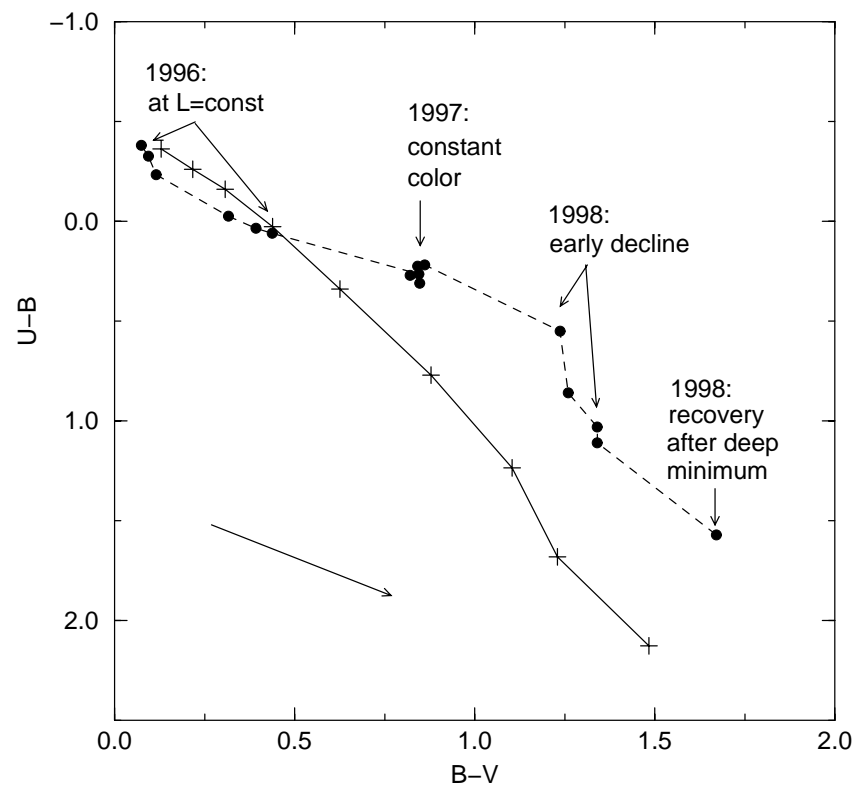

Fig. 5. $-U-B$ vs. $B-V$ diagram of V4334 Sgr, 1996-1998, dereddened for the value $E_{B-V}=0.8$ of interstellar reddening. The long arrow at the lower left shows the slope of the interstellar reddening line $E_{U-B} / E_{B-V}$. Important stages during 1996, 1997, and 1998 are marked by arrows. In the maximum stage of 1996-1997, the pseudo photosphere cooled at constant luminosity ("at $\mathrm{L}=$ const"), but this cooling process seems to have come to a halt in 1997 ("constant color"). The dust onset stage in early 1998 and the beginning of the massive dust stage in late 1998 are labeled as "early decline." For the following brightness evolution, $U$ data are missing, except for a point taken during a temporary recovery of brightness in very late 1998 ("recovery after deep minimum"). The solid line marks the locus of hydrogen-deficient stellar atmospheres calculated by M. Asplund (1997, private communication), where the effective temperatures of 9000,8500 , $8000,7500,7000,6500$, and $6000 \mathrm{~K}$ are marked by plus signs.

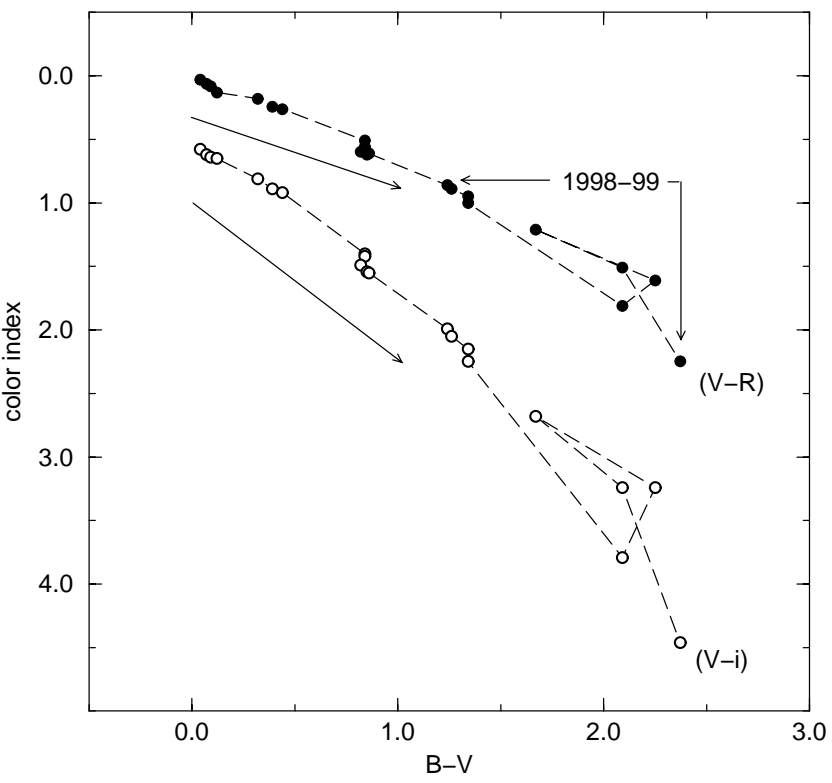

FIG. 6. $-V-R$ vs. $B-V$ (filled circles) and the $V-i$ vs. $B-V$ (open circles) paths of V4334 Sgr, 1996-1998, dereddened for $E_{B-V}=0.8$. The two arrows in the upper left corner show the slopes of the interstellar reddening lines $E_{V-R} / E_{B-V}$ (top arrow) and $E_{V-i} / E_{B-V}$ (bottom arrow). The $V-i$ data points have been shifted by $+0.5 \mathrm{mag}$ for clarity. The figure shows the gradual reddening of 1996, the stagnation of 1997, the subsequent dust formation of early 1998, as well as the loop of 1998-1999, marking the second fading, the subsequent recovery, and the third fading.

indices for about 150 days, indicating that the photosphere had become stationary. In the case of a pseudo photosphere, whose location is determined by the actual massloss rate, this means that the mass-loss rate had stabilized.

TABLE 3

Color Evolution of V4334 SagitTariI

\begin{tabular}{ccccccc}
\hline \hline JD & $V$ & $U-B$ & $B-V$ & $V-R$ & $V-i$ & Reference \\
\hline $2,450,136 \ldots \ldots$ & 11.29 & $\ldots$ & 0.84 & 0.53 & 1.11 & $\ldots$ \\
$2,450,144 \ldots \ldots$ & 11.22 & 0.23 & 0.87 & 0.56 & 1.15 & $\ldots$ \\
$2,450,173 \ldots \ldots$ & 11.15 & 0.28 & 0.89 & 0.58 & 1.17 & $\ldots$ \\
$2,450,196 \ldots \ldots$ & 11.06 & 0.38 & 0.92 & 0.63 & 1.18 & $\ldots$ \\
$2,450,287 \ldots \ldots$ & 11.03 & 0.58 & 1.12 & 0.68 & 1.34 & $\ldots$ \\
$2,450,328 \ldots \ldots$ & 10.91 & 0.64 & 1.19 & 0.74 & 1.42 & $\ldots$ \\
$2,450,350 \ldots \ldots$ & 10.86 & 0.67 & 1.24 & 0.76 & 1.45 & $\ldots$ \\
$2,450,550 \ldots \ldots$ & 10.88 & 0.87 & 1.64 & 1.01 & 1.93 & $\ldots$ \\
$2,450,595 \ldots \ldots$ & 10.98 & 0.83 & 1.64 & 1.06 & 1.95 & $\ldots$ \\
$2,450,611 \ldots \ldots$ & 10.96 & 0.88 & 1.62 & 1.10 & 2.02 & $\ldots$ \\
$2,450,684 \ldots \ldots$ & 10.85 & 0.92 & 1.65 & 1.12 & 2.07 & $\ldots$ \\
$2,450,706 \ldots \ldots$ & 10.94 & 0.83 & 1.66 & 1.11 & 2.08 & $\ldots$ \\
$2,450,895 \ldots \ldots$ & 12.21 & 1.16 & 2.04 & 1.36 & 2.52 & $\ldots$ \\
$2,451,042 \ldots \ldots$ & 12.66 & 1.47 & 2.06 & 1.39 & 2.58 & $\ldots$ \\
$2,451,059 \ldots \ldots$ & 13.22 & 1.64 & 2.14 & 1.45 & 2.68 & $\ldots$ \\
$2,451,070 \ldots \ldots$ & 13.92 & 1.72 & 2.14 & 1.50 & 2.78 & $\ldots$ \\
$2,451,094 \ldots \ldots$ & 18.09 & $\ldots$ & 2.89 & 2.31 & 4.32 & JDM \\
$2,451,105 \ldots \ldots$ & 16.91 & $\ldots$ & 3.05 & 2.11 & 3.77 & JDM \\
$2,451,126 \ldots \ldots$ & 15.14 & 2.18 & 2.47 & 1.71 & 3.21 & $\ldots$ \\
$2,451,237 \ldots \ldots$ & 16.85 & $\ldots$ & 2.89 & 2.01 & 3.77 & $\ldots$ \\
$2,451,259 \ldots \ldots$ & 20.08 & $\ldots$ & 3.17 & 2.75 & 4.99 & J \\
$2,451,267 \ldots \ldots$ & 20.08 & $\ldots$ & $\ldots$ & 2.36 & 4.52 & $\ldots$ \\
$2,451,368 \ldots \ldots$ & 22.10 & $\ldots$ & $\ldots$ & 1.23 & 3.59 & TNG \\
\hline
\end{tabular}

NotE.-JDM = Jacoby $\quad \& \quad$ De $\quad$ Marco $\quad$ (1998); J = Jacoby $\quad$ (1999); TGN $=$ Telescopio Nazionale Galilei (this paper). 


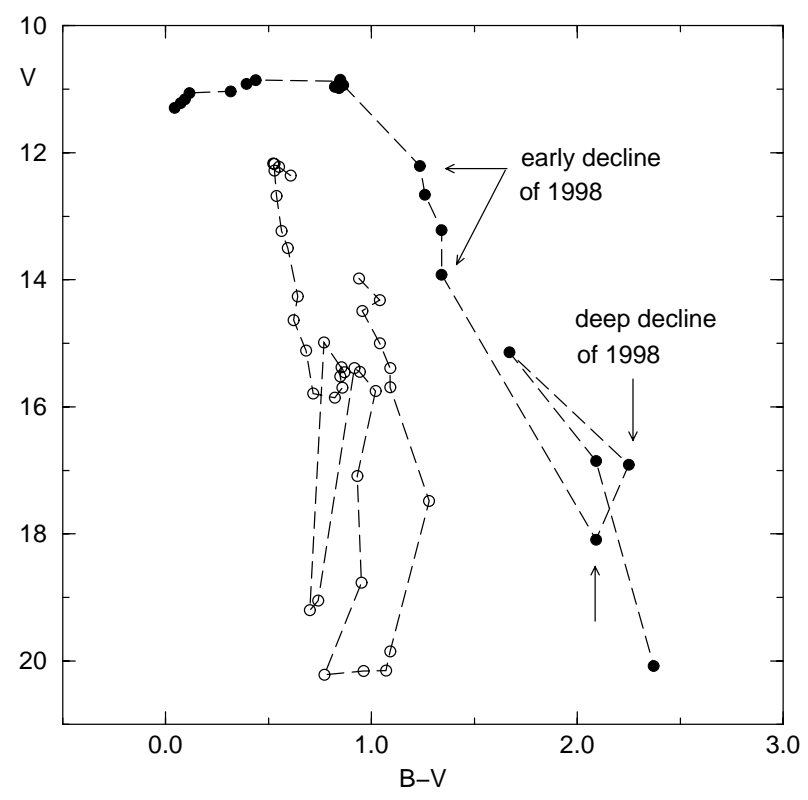

FIG. 7. $-V$ vs. $B-V$ diagram of V4334 Sgr. The $B-V$ values are dereddened for $E_{B-V}=0.8$. Two points are available for the deep (second) decline of 1998, when the $V$ magnitude reached about $19 \mathrm{mag}$; they are marked by arrows. After the brightness recovery in late 1998 and early 1999, V4334 Sgr dropped to fainter magnitudes and somewhat redder colors. The declines in brightness in 1998 and 1999 were similar to the red declines of R CrB stars. For comparison, the deep decline of 1991 of the R CrB star V854 Cen, which is also composed of several declines and recoveries, is shown (open circles; the $V$ magnitudes were shifted by 5 mag to fit into the diagram; data were taken from Lawson et al. 1992).

During the dust onset and the massive dust stages, the colors show a reddening of increasing strength, while the visible light of V4334 Sgr declined. The reddening increased noticeably in the deep decline of early 1998 October, which is documented by observations of Jacoby \& De Marco (1998). Since no $U$ magnitudes were reported, this episode is missing from Figure 5 and is only illustrated in the other two-color diagrams (Fig. 6) and in the $(V, B-V)$ colormagnitude diagram (Fig. 7). In 1999 the star had become so faint in $U$ that no observations were obtained. For 1999 July an upper limit of $U=23.5 \mathrm{mag}$ is derived, but the previously recorded color indices make it likely that the star was several magnitudes fainter. Our $V$ observations show fluctuations around 19.5-20 mag in 1999 March and a minimum brightness of $22.1 \mathrm{mag}$ in $1999 \mathrm{July}$. Note that the very last $V-R$ and $V-i$ indices of 1999 , which are not included in the figures because of the lack of $B$ magnitudes, indicate a decrease in reddening in spite of the faintness of the star (see also $\S 5$ ).

\section{DUST-FORMING EVENTS AS SEEN IN THE OPTICAL REGION}

Theoretical, spectroscopic, and photometric results have pointed out a possible relation between $\mathrm{R}$ CrB stars and final He flash objects such as FG Sge (Gonzalez et al. 1998; Jurcsik \& Montesinos 1999) and V4334 Sgr (Asplund et al. 1997; Arkhipova et al. 1999). Dust-forming events, similar to those seen in $\mathrm{R} \mathrm{CrB}$ stars, have been suspected in the light curve of the rapidly evolving final He flash object V605 Aql (Harrison 1996), and they have been observed in recent years in the slowly evolving final He flash object FG Sge. Already in the first papers on V4334 Sgr, a dust-forming phase was predicted (D96; Duerbeck \& Pollacco 1996). The declines observed in V4334 Sgr (and first announced by Liller et al. 1998a, 1998b) can readily be compared with those observed in the other well-observed final He flash object, FG Sge, and in R CrB-type variables. Fading events of R CrB and V854 Cen, observed by Cottrell, Lawson, \& Buchhorn (1990), Lawson \& Cottrell (1989), and Lawson et al. (1992), using multicolor photometry, were useful in comparing photometric and spectroscopic characteristics of $\mathrm{He}$ flash objects and $\mathrm{R} \mathrm{CrB}$ variables.

\subsection{Pulsations and Declines}

$\mathrm{R} \mathrm{CrB}$ and related hydrogen-deficient stars show pulsations at maximum light. Periods are 40-100 days, with amplitudes of a few $0.1 \mathrm{mag}$. A tendency toward longer periods in cooler objects is seen (Lawson et al. 1990). In the $\mathrm{R}$ CrB star V854 Cen, the onset of a brightness decline usually occurs near maximum light of the pulsation cycle (Lawson et al. 1992), while in RY Sgr it occurs at minimum light (Menzies \& Feast 1997). The slowly evolving final He flash object FG Sge has shown pulsation periods ranging from 5 to 138 days, with a definitive trend to longer periods at later times, when the object had cooled (van Genderen \& Gautschy 1995). After the first steep decline in 1992, FG Sge showed a nearly constant pulsation period of 115 days, and $\mathrm{R}$ CrB-type fadings often occurred near maximum light of the pulsation (Gonzalez et al. 1998).

V4334 Sgr showed variations with cycles of $\approx 10-74$ days, with a tendency toward better defined, longer periods at later (cooler) stages and amplitudes increasing from 0.1 to 0.7 mag. Apart from the rapid change of behavior, the pulsations of V4334 Sgr are comparable with those of R CrB stars, as well as with the pulsations of FG Sge. The three observed fading events of V4334 Sgr are separated by intervals of $\sim 200$ days, and do not seem to be related to the period of stellar pulsation. The well-documented second fading of 1998 September began around a minimum phase of pulsation.

\subsection{Color Evolution During Declines}

$\mathrm{R}$ CrB stars show both "blue" and "red" declines (Cottrell et al. 1990). The blue events presumably occur when the obscuring cloud is smaller than the photosphere, as seen from the observer. In a red decline of $\mathrm{R} \mathrm{CrB}$, the star moved along the line $(U-B) /(B-V) \approx 1$ in the two-color diagram. A well-observed red decline of V854 Cen had $(U-B) /(B-V) \approx 0.6, \quad(V-R) /(B-V) \approx 0.8, \quad(V-I) /$ $(B-V) \gtrsim 1.6$. The slowly evolving final He flash object FG Sge has only shown blue declines (Jurcsik \& Montesinos 1999). Thus, dust formation in FG Sge has been patchy until now. The color behavior of V4334 Sgr is complex, because the growth of the photosphere, the dust formation, and even the interstellar reddening cause similar effects in two-color diagrams. While interstellar reddening just produces a constant shift in the diagram, the first two effects can most easily be disentangled with the aid of the $V$ versus $B-V$ diagram (Fig. 7). It shows that the initial dust formation episode in the line of sight did not begin until early 1998.

V4334 Sgr shows a "composite" of several red declines, indicating that in all cases the whole visible photosphere is obscured (Fig. 2). The first decline occurred at or before JD 2,450,853 (1998 February), and the star did not regain its former brightness; the second occurred around 
JD 2,451,045 (1998 September), and the star partly recovered; the third one occurred at or before JD 2,451,234 (1999 February), and, after some fluctuations at $20 \mathrm{mag}$, another decline followed, whose color characteristics are only poorly known. The value of $(U-B) /(B-V)$ appears always to be larger than it is in the case of $\mathrm{R} \mathrm{CrB}$ stars. Following the onset of dust formation in 1998 February, a sequence of three normal points indicates the beginning of the fading of 1998 September (marked "early decline" in Fig. 5). After the deep decline in 1998 October and the recovery in November, which was observed by Jacoby \& De Marco (1998), another $U-B$ color index is available (marked "recovery after deep minimum"). The overall slope of the $1998(U-B) /(B-V)$ data is $\approx 2.4$. This slope is substantially steeper than the slopes $\approx 1$ and $\approx 0.6$ observed in $\mathrm{R}$ $\mathrm{CrB}$ and V854 Cen. Whether this is caused by different dust properties or a different amount of "chromospheric emission" above the dust in these objects cannot be decided on the basis of the available data.

The other color index ratios, $(V-R) /(B-V)=1.0$, $(V-i) /(B-V) \approx 1.8$, derived for the interval 1998-1999 when dust obscuration was obviously present, are surprisingly similar to those observed in V854 Cen (and cum grano salis to the interstellar reddening lines), $(V-R) /$ $(B-V)=0.8,(V-I) /(B-V)=1.6$. Both V854 Cen and V4334 Sgr show a tendency to yield steeper slopes at very red colors and very faint magnitudes.

Even during a red decline, all color indices of V854 Cen turn to smaller values at very faint magnitudes. The fragmentary data of V4334 Sgr indicate that a similar effect occurred in $1999 \mathrm{July}$, when the $V$ magnitude reached its observed minimum near $22 \mathrm{mag}$, and the $V-R$ and $V-i$ indices were noticeably smaller than three months before. Whether this is also the signature of an imminent brightness recovery, as it is found in $\mathrm{R} \mathrm{CrB}$ stars, cannot be said because of lack of data.

\subsection{Depth and Speed of Declines}

The deepest decline in $\mathrm{R} \mathrm{CrB}$ or related stars ever observed in the visible region was 8 mag (see the light curve of R CrB by Mattei, Waagen, \& Foster 1991). Such a level may be reached during a single fading event or by a superposition of several fading events. In the latter case the star does not become fainter but may simply remain for a longer time at minimum level. Concerning the speed of declines, a red decline of R CrB observed by Fernie, Percy, \& Richer (1986) showed a maximum rate of decline of $0.13 \mathrm{mag}$ day $^{-1}$ in its later stages; a rate of 0.27 mag day ${ }^{-1}$ was observed by Cottrell et al. (1990) during a blue decline. During the deep red decline of V854 Cen in 1991, with a superposition of three fading events, gradients up to 0.7 mag day ${ }^{-1}$ were observed (Lawson et al. 1992).

During 1998-1999, V4334 Sgr declined by 11 mag in $V$, i.e., the obscuration was at least an order of magnitude more efficient than ever observed for an $\mathrm{R} \mathrm{CrB}$ star. This decline consists of several superposed fading events, as described in $\S 5.2$. The object partially recovered from the first two events; the sparse data of 1999 indicate that V4334 Sgr is still obscured by the third fading event (it is also possible that a fourth fading event took place). The first decline was not covered by observations, the decline rates of the second and third declines were $0.05 \mathrm{mag} \mathrm{day}^{-1}$ and 0.14 mag day ${ }^{-1}$. The speed and form of these declines resemble those of slow red declines of $\mathrm{R} \mathrm{CrB}$ variables.

\subsection{Spectroscopic Features of Dust Shells}

Dust that forms near an $\mathrm{R} \mathrm{CrB}$ star experiences a strong radiation force, moves outward, and drags gas with it, which is collisionally excited. $\mathrm{R} \mathrm{CrB}$, while recovering from a decline and at subsequent maximum light, showed a $P$ Cyg line of He I 10830 extending to $-240 \mathrm{~km} \mathrm{~s}^{-1}$ (Querci \& Querci 1978). In spectra of V4334 Sgr, a blueshifted absorption line of $\mathrm{He}$ I 10830 with an expansion velocity of -550 $\mathrm{km} \mathrm{s}^{-1}$ was observed in 1998 March by Eyres et al. (1999), i.e., shortly after the onset of dust formation in the line of sight. No line had been present in spectra taken in 1997 July. From 1998 August onward, the line shape changed to a P Cygni profile (Eyres et al. 1999; Tyne et al. 2000). During the deep minimum of 1999, the $\mathrm{He}$ I line was observed in emission only, and it extended less to the red than the P Cyg line of the previous year. It showed a blueshift of about $-500 \mathrm{~km} \mathrm{~s}^{-1}$ relative to the radial velocity of V4334 Sgr (limits -700 and $+130 \mathrm{~km} \mathrm{~s}^{-1}$ ). This indicates that (1) collisionally excited gas has existed since early 1998 , in agreement with the photometrically observed onset of dust formation; (2) while the stellar background faded, the line kept its strength and appeared as a P Cyg line in a semitransparent shell; and (3) after the massive dust formation, emission originating in the region moving away from the observer is almost completely obscured by dust, and only the emission originating in the hemisphere facing the observer is seen.

\subsection{Infrared Behavior}

The infrared behavior of $\mathrm{R} \mathrm{CrB}$ stars correlates poorly with dust-forming events in the line of sight, which mainly influence the flux in the optical region. Since the dust cloud is small, it converts only a small fraction of the total light of the star into infrared radiation. The infrared output of an $R$ $\mathrm{CrB}$ star is dominated by radiation from the overall circumstellar dust shell, which is heated by the star (and also shows the pulsational light variations observed in the star). On the other hand, the dust flux does not change significantly when the star goes into an obscuration minimum. These findings are the best evidence for the patchiness of dust formation in the atmospheres of R CrB stars (Forrest, Gillett, \& Stein 1972; Feast et al. 1997). The descent to minimum in V4334 Sgr was accompanied by a complete change in its energy distribution, including a dramatic increase at infrared wavelengths (especially in the poorly observed range $\lambda>5 \mu \mathrm{m}$ ). A more detailed study of the steady growth of the infrared excess is given in $\S 6$.

\subsection{V4334 Sgr and R CrB Stars: Concluding Remarks}

Summing up, the fading events of V4334 Sgr in the years 1998-1999 show striking similarities with the red declines of $\mathrm{R}$ CrB stars. While there are differences between individual stars in the $U-B / B-V$ ratio, all objects show a similar behavior at longer wavelengths. In contrast to $\mathrm{R} \mathrm{CrB}$ stars, the unusually deep, long-lasting minimum, the evolution of the He I 10830 line structure, as well as the connection between optical fading and infrared brightening, indicate the formation of a complete dust shell around V4334 Sgr. The behavior of V4334 Sgr in 1998 and later should not necessarily be described as the " $\mathrm{R}$ CrB phase," as Arkhipova et al. (1999) have done. R CrB stars form no complete dust shells. Its behavior also shows striking similarities with dust-forming classical novae, as will be shown in $\S 6.2$. 


\section{ENERGY DISTRIBUTION AND LUMINOSITY}

The $U B V R i$ photometry presented in this study can be combined with infrared photometry to a study of the overall energy distribution, the character of the infrared excess, and the time variation of the luminosity. Infrared photometry is available during each season (M. Feast \& P. A. Whitelock 1999, private communication) for 20 well-distributed $J H K L$ data sets obtained between early 1996 to late 1999; Kamath \& Ashok (1999) for 1996 and 1997 in JHK; Fouqué (in D96) for 1996 April in IJK; Arkhipova et al. (1998) for 1996 and 1997 in JHKLM; Kimeswenger et al. (1997) for 1997 March in IJK; Kerber et al. (1999) for 1997 and 1998 at seven wavelengths between 4.5 and $12.0 \mu \mathrm{m}$, observed with ISOCAM of the Infrared Space Observatory (ISO); Lynch et al. (1998) for 1998 March and May in $L^{\prime}, M^{\prime}, N^{\prime}$; Kaeufl \& Stecklum (1998) for 1998 June in N; Jacoby (1999) for 1999 April at 1.083 and $2.230 \mu \mathrm{m}$; Tyne et al. (2000) for 1999 April and May in JHKLM; Hinkle \& Joyce (1999) for 1999 September in JHKLM.

A selection from these data was combined with quasisimultaneous $U B V R i$ data to construct 21 energy distributions of V4334 Sgr: seven for 1996, seven for 1997, three for 1998 , and four for 1999. The magnitudes were dereddened for the value $E_{B-V}=0.8$ and were converted into monochromatic irradiances $E_{\lambda}$ (in $\mathrm{W} \mathrm{m}^{-2} \mu \mathrm{m}^{-1}$ ). They are listed in Table 4. Integration over the irradiances $E_{\lambda}$ yielded total irradiances (in $\mathrm{W} \mathrm{m}^{-2}$ ), which are also given. Selected results are shown in Figure 8.

\subsection{Evolution of the Infrared Excess}

Figure 8 shows that an excess of radiation at wavelengths $>1 \mu \mathrm{m}$ already exists during the earliest observations of 1996. This excess increases in strength in 1997 and starts to dominate the spectrum in 1998. The stellar continuum peaks at $B$ in 1996, at $V$ in 1997, between $V$ and $R$ in 1998, and possibly at $R$ in 1999 . From 1998 onward, the optical

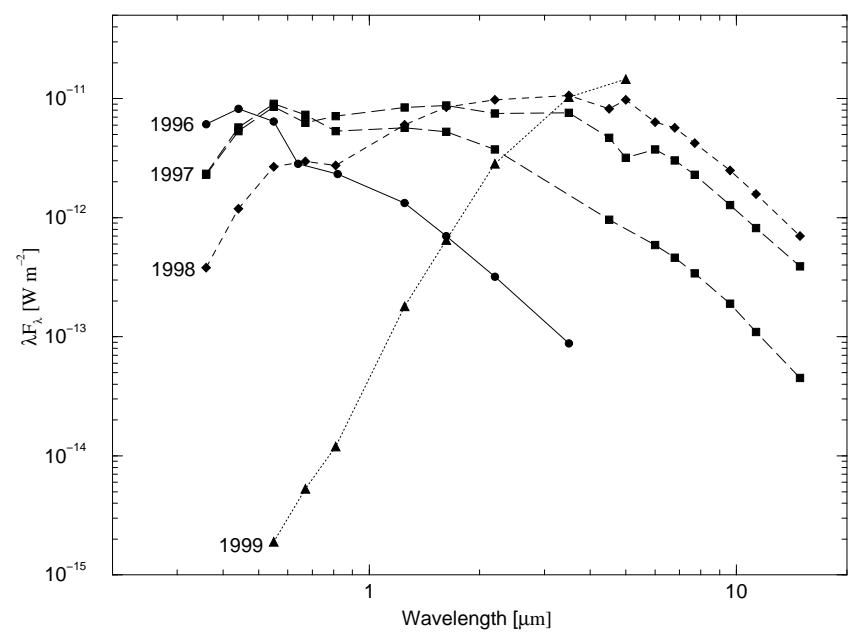

FIG. 8.-Selected energy distributions of V4334 Sgr between 1996 and 1999. 1996 March (circles connected by lines); 1997 February and September (squares connected by long dashed lines); 1998 March (diamonds connected by dashed lines); 1999 May (triangles connected by dotted lines). The stellar continuum curve is easily recognized. Its maximum shifts between 1996 and 1997 toward longer wavelengths; between 1997 and 1998 such an effect is less noticeable. Note that an infrared excess is always present and that it increases in strength with time. By 1998 it starts to influence the short-wavelength-region data, while in 1999 hardly any indication of the presence of the optical stellar continuum has remained. radiation of the star is extinguished and reradiated at infrared wavelengths. In 1999, hardly a trace of the stellar contribution is visible in the energy distribution.

A preliminary discussion of the infrared energy distributions is given by Kipper (1999). Several test runs using DUSTY, ${ }^{7}$ with parameters similar to those chosen by Kipper, yielded nonoptimal fits, which can possibly be explained by the different adopted value of the interstellar extinction and the different choice of the stellar atmosphere. A detailed analysis of the spectral energy distribution will be the subject of a future investigation.

Figure 8 permits estimates of the properties of the infrared excess, the character of the dust, and the size of the dust-forming region, and some qualitative estimates will be given. The radiation maximum of the infrared excess shifts toward longer wavelengths at later times. If the excess is approximated by a blackbody, its temperatures are $\sim 3500$, $3000 \rightarrow 2000,830$, and $725 \mathrm{~K}$ in 1996, 1997, 1998, and 1999, respectively. The temperatures of 1996 and 1997 are too high for the formation of carbon dust.

The infrared excess of 1996 may be explained by free-free emission in the wind or expanding atmosphere of V4334 Sgr, which at that time had a surface temperature of $\sim 7500$ K. In 1997 the situation is not as clear. Woitke, Goeres, \& Sedlmayr (1996) have shown that carbon nucleation can take place in shocks that occur in pulsating $\mathrm{R} \mathrm{CrB}$ stars with effective surface temperatures of $7000 \mathrm{~K}$. Since V4334 Sgr provides similar conditions, patchy dust formation may be possible in 1997. From 1998 onward, observational evidence of dust formation is beyond doubt, as was shown in $\S 5.2$. The angular radius of the infrared emission region, calculated from $\theta=2 \times 10^{12}\left(\lambda F_{\lambda}\right)_{\max }^{1 / 2} T^{-2}$ (Gallagher \& Ney 1976) with $\theta$ in milliarcseconds and $\left(\lambda F_{\lambda}\right)_{\max }$ in $\mathrm{W} \mathrm{m}^{-2}$, is $0.3,0.8,9.4$, and 11.4 mas for the years $1996-1999$, respectively. Figure 9 indicates that a rapid growth of the dust shell occurred between late 1997 and early 1998 .

Assuming a distance of $2 \mathrm{kpc}$ for the object, the radius of the dust shell was about $19 \mathrm{AU}$ in 1998 and $23 \mathrm{AU}$ in 1999. If the ejection of material started in early 1995, at the time of

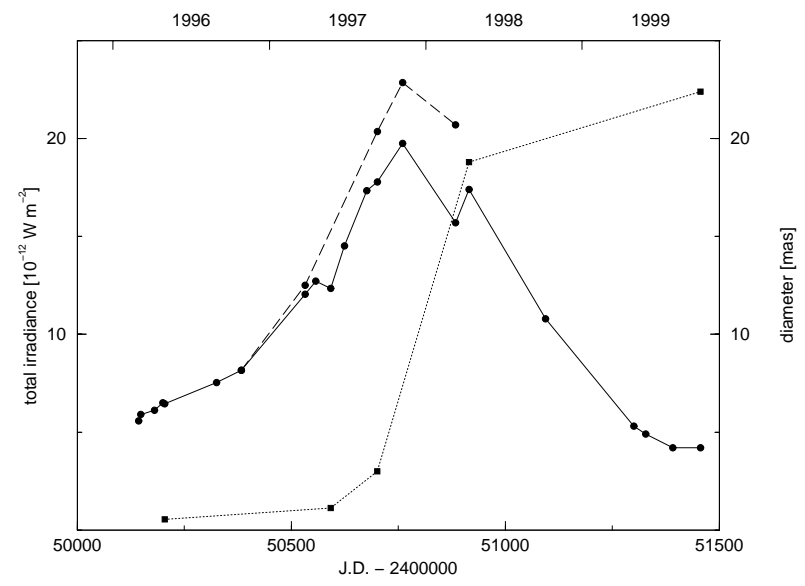

FIG. 9.- Temporal evolution of the luminosity of V4334 Sgr and the diameter of its radiating dust shell. The circles connected by solid lines give the total irradiance shortward of $4.5 \mu \mathrm{m}$; the circles connected with long dashes give the total irradiance up to $14.9 \mu \mathrm{m}$ (ISO data). The squares connected by dots give the diameter of the dust shell in milliarcseconds.

\footnotetext{
${ }^{7}$ See User Manual for DUSTY at http://www.pa.uky.edu/moshe/ dusty/manual.html.
} 


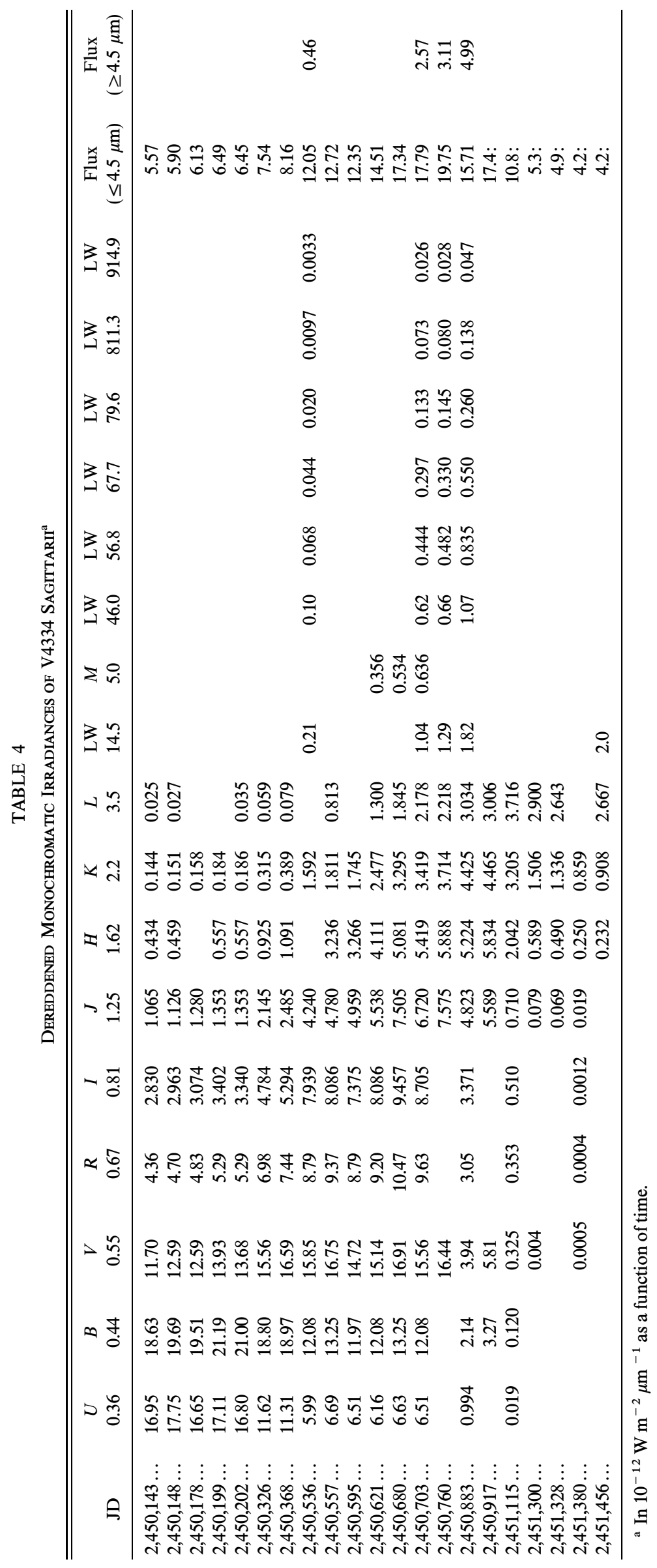


the beginning of the final He flash, and if dust had condensed everywhere in the shell in mid-1998, the constant expansion velocity of $25 \mathrm{~km} \mathrm{~s}^{-1}$ of dust-forming material is derived. Between mid-1998 and mid-1999 the shell grew with a linear velocity of $20 \mathrm{~km} \mathrm{~s}^{-1}$, which is in good agreement with the previous value. This velocity is much larger than the rate of growth of the photosphere (about $1 \mathrm{~km}$ $\mathrm{s}^{-1}$ ), which was derived from the observations of 1996 (D97). Thus we may take it as evidence for a Eddingtondriven outflow above the photosphere, which had been active since the beginning of the final helium flash and had cooled to temperatures suitable for dust formation around the end of 1997. On the other hand, we can assume that the material was ejected at a later phase of the outburst, when the outer layers had already been enriched with carbon, and that the condensing dust experienced an acceleration due to radiation pressure. Then the resulting expansion velocity is higher, and it may be similar to the velocity of the central condensations of the remnant of V605 Aql $\left(100 \mathrm{~km} \mathrm{~s}^{-1}\right.$ with a FWHM of $225 \mathrm{~km} \mathrm{~s}^{-1}$; Pollacco et al. 1992). More observations are necessary to decide between the two scenarios.

\subsection{Infrared Evolution of V4334 Sgr and in Classical Novae}

Final He flash objects and R CrB-type stars have similar properties, as described in $\S 5$. Similarities, however, also exist between final He flash objects and dust-forming classical novae. The infrared behavior of dust-forming classical novae consists of four phases: (1) the initial pseudo photosphere blackbody, (2) a free-free phase, which leads to an infrared excess between 1-6 $\mu \mathrm{m}$, (3) a rapid growth of dust, leading to a red decline in the optical and an increase of infrared flux and of angular diameter, and a slight drop in dust temperature $(1200 \rightarrow 800 \mathrm{~K})$, and (4) an exponential drop of the infrared flux and of the angular diameter, when dust is being dispersed and/or destroyed by radiation from the central source, accompanied by a recovery of UV and optical radiation (Ney \& Hatfield 1978).

In 1996 and 1997 the derived blackbody temperature for the infrared excess of V4334 Sgr is too high for dust formation; it was already pointed out that the infrared excess of 1996 (and possibly 1997) is caused by free-free emission in the outflowing material that had passed the pseudo photosphere and formed an extended atmosphere. Claims of the presence of dust with temperatures of 1500,1800 , and 680 K, in 1997 February, March, and April (Kerber et al. 1999; Kimeswenger et al. 1997; Eyres et al. 1998a) are questionable and discrepant. Part of the infrared excess may be carbon nucleation products; part of it may still be explained by free-free continuum emission.

At the end of 1997 or the beginning of 1998, the temperature of the extended atmosphere had dropped below $2000 \mathrm{~K}$, permitting the formation of carbon dust. This onset of dust formation led to (1) a rapid growth of the angular size of the infrared emitting region (Fig. 9), (2) an increase of the infrared flux, and (3) several dust-forming events in the line of sight, which caused first a gentle, then a dramatic, drop in visible light output $(\$ 5.2)$.

Comparing the evolution of V4334 Sgr with that of a dust-forming classical nova, e.g., NQ Vul (Ney \& Hatfield 1978) yields the following: Phase 1 likely occurred in 1995 but was not observed; phase 2 occurred in 1996-1997; and phase 3 in 1998-1999. The onset of phase 4, the destruction or dispersion of the dust, may still be far in the future. Eighty years after outburst, the central object of V605 Aql is still deeply embedded in circumstellar dust, and we can expect a similar behavior for V4334 Sgr.

The "speed" of a classical nova like NQ Vul to evolve through phases 1 to 3 is about 20 times faster than that of the final He flash object V4334 Sgr. One more noteworthy difference between a nova and a final He flash object exists. The spectrum emerging from the pseudo photosphere of the nova near maximum light clearly reveals the speed of the outflowing material, and the expansion rate of the infrared dust shell (in milliarcseconds per day) can be used to determine the shell expansion parallax. High-resolution spectra of V4334 Sgr described by D97, Kipper \& Klochkova (1997), and Jacoby et al. (1998) show that the radial velocity of the photosphere is similar to that of the planetary nebula. In an echelle spectrum, however, taken 1996 April 23 by G. Wallerstein, the deep $\mathrm{H} \alpha$ absorption line shows an underlying shallow, broad absorption trough ranging from -225 to $+170 \mathrm{~km} \mathrm{~s}^{-1}$ relative to the star. This may be taken as evidence for optically thin, turbulent material, which shows an average outflowing velocity of $\sim 25 \mathrm{~km} \mathrm{~s}^{-1}$, with a wide spread in velocities. A careful study of spectral features of the wind, in combination with data of the growth of the dust shell, may permit the derivation of a shell expansion parallax.

Dust-forming novae show optically thick winds with high outflow velocities that exist on timescales of weeks; V4334 Sgr shows an optically thin wind with an outflow velocity of $25 \mathrm{~km} \mathrm{~s}^{-1}$ (or several times higher), which likely exists during all stages of the outburst. The increase of luminosity at later stages ( $(6.3)$, in combination with the cooling of the outer layers, may lead to a enhanced mass loss at later times.

\subsection{Variations in Luminosity and Limits on the Mass of V4334 Sgr}

The data of Table 4 can be used to study the luminosity of V4334 Sgr at various stages of its evolution. The total irradiances (in units of $10^{-12} \mathrm{~W} \mathrm{~m}^{-2}$ ) are also shown in Figure 9. From 1997 onward, an ever increasing part of the luminosity is radiated at wavelengths longward of $4.5 \mu \mathrm{m}$, and nothing quantitative can be said about the luminosity evolution after early 1998 because far-infrared data are lacking.

The flux increased by a factor of 4 from early 1996 to early 1998. This result depends only weakly on the assumed value of the interstellar extinction. It was already noted by D97 that the assumption of a constant luminosity of V4334 Sgr was not valid for the 1996-1997 light curve; the flux in the optical region increased by at least $30 \%$ over $1 \mathrm{yr}$. A possible explanation of this behavior is that, in early stages of the flash, a significant fraction of the energy release is used for the expansion of the object (this is also shown, implicitly, in theoretical tracks of final He flash objects; e.g., Figs. 14 and 15 in Blöcker 1995).

We take the "late" 1997-1998 luminosity of V4334 Sgr as the luminosity emerging from the remnant after most of the expansional work had been done. A total irradiance of $2.2 \times 10^{-11} \mathrm{~W} \mathrm{~m}^{-2}$ is assigned to V4334 Sgr. This can easily be converted into a radiant flux release of $10^{30}(\mathrm{~d} / 2$ $\mathrm{kpc})^{2} \mathrm{~W}$ or

$$
L_{\mathrm{V} 4334 \mathrm{Sgr}} \sim 2770 L_{\odot}\left(\frac{d}{2 \mathrm{kpc}}\right)^{2} .
$$


A high-mass post-AGB model of Blöcker (1995) has a mass of $0.836 M_{\odot}$ and takes $50 \mathrm{yr}$ to make its way from the planetary nebula nucleus back to the AGB during the final He flash; its mass may be taken as a lower limit to the mass of V4334 Sgr. The luminosity of the model is $20,000 L_{\odot}$, which may serve as a reasonable lower limit to the luminosity and thus to the distance of V4334 Sgr. Insertion in the above relation yields $5.4 \mathrm{kpc}$ for V4334 Sgr. Even lowmass models of $\sim 0.6 M_{\odot}$ yield distances $>2 \mathrm{kpc}$, and the assumption of $\S 3, d>2 \mathrm{kpc}$, is always fulfilled. If one would use the range of absolute $B$ magnitudes of central stars of planetary nebulae as a way to estimate the mass of V4334 Sgr, the upper distance limit of $3 \mathrm{kpc}$ yields a mass slightly above $0.6 M_{\odot}$, which is somewhat unlikely in view of its fast evolution (see below and § 7). Diagrams showing evolutionary speeds and envelope and core masses for born-again giants, as given by Blöcker \& Schönberner (1997), do not cover the rapid evolution of V4334 Sgr. Nevertheless, an envelope mass of $10^{-5} M_{\odot}$ and a core mass around $1 M_{\odot}$ are reasonable guesses. The high luminosity of such an object may even give support to the "long" distance scale of $8 \mathrm{kpc}$ as suggested by D97. Further research on post-AGB evolution is clearly needed to constrain the distance of V4334 Sgr.

\section{TIMESCALES}

Data exist on the evolution of two previous final He flash objects, FG Sge and V605 Aql. We omit from our discussion the seventeenth century object CK Vul, whose nature is still not clear and whose light curve covers only the brightest stages (Harrison 1996). Data were taken from Harrison (1996), Clayton \& De Marco (1997), and Jurcsik \& Montesinos (1999) and compared with the present data. Table 5 gives the timescales involved.

The only existing high-quality spectrum of V605 Aql was described by Bidelman (1973) to be "very similar to the hydrogen-deficient carbon star HD 182040," which has a type C2, 2 in the old Keenan-Morgan classification, and C-HD1C $4_{2}{ }^{-}$CH0 in the 1993 revised MK system of Keenan (Barnbaum, Stone, \& Keenan 1996). The appearance of the spectrum of V4334 Sgr, taken in May 1997 and analyzed by Pavlenko, Yakovina, \& Duerbeck (2000) is strikingly similar to that of V605 Aql, as illustrated by Clayton \& De Marco (1997) and classified by Bidelman.

We compare the light curves of FG Sge, V605 Aql, and V4334 Sgr in detail, taking the light curve of Harrison (1996) for V605 Aql and assuming that maximum $B$ (or photographic) light occurred in 1968, 1919.6, and 1996.3 for the three objects, respectively. Note that Harrison's light curve of V605 Aql already shows a minimum in 1920; this "first" dust event, however, seems to be poorly documented and will not be taken into consideration here. The following time intervals are derived. Rise from about 15 mag to $B$ (or photographic) maximum took $74,1.9$, and $1.5 \mathrm{yr}$, respectively. A comparable spectral type C2, 2 was reached about 20, 2.1, and $1.0 \mathrm{yr}$ after maximum. Dust event onset, first dust event, and first minor dust event were observed 24, 3, and $2.1 \mathrm{yr}$ after maximum. "Disappearance" due to a major dust event has not yet been observed for FG Sge and occurred 4.4 and $2.9 \mathrm{yr}$ after maximum for the two other objects. The "total duration of visibility" (with moderate means) is thus 6.5 and $4.4 \mathrm{yr}$ for V605 Aql and V4334 Sgr, respectively. FG Sge has not yet entered the stage of faintness, and one can only compare the time from the onset of brightening with the onset of dust formation, which is $98 \mathrm{yr}$ for FG Sge, $4.9 \mathrm{yr}$ for V605 Aql, and $3.6 \mathrm{yr}$ for V4334 Sgr. "Averaging" the timescales of various events in the three objects, one finds that V4334 Sgr is the most rapidly evolving (and presumably the most massive) final He flash object known; V605 Aql is about 50\% slower, and FG Sge is a factor of 25-50 slower. Extrapolating the lifetime of FG Sge to its expected disappearance due to a future major dust event yields a value of up to $220 \mathrm{yr}$. Already one-half of this time has elapsed; it will be interesting to monitor the future evolution of FG Sge.

\section{SUMMARY AND OUTLOOK}

The complete multicolor light curve of V4334 Sgr from its prediscovery rise to the dust obscuration shows that the color indices increase quite smoothly. In 1995-1997, this is caused by the cooling of the expanding pseudo photosphere of a mass-losing object that has a slowly increasing luminosity. Furthermore, the increasing infrared excess can be explained by free-free emission in an Eddington-driven outflow. Starting from 1998, brightness drops and their color characteristics mimic the red declines of $\mathrm{R} \mathrm{CrB}$ variables. The increase in infrared flux and the behavior of the collisionally excited $\mathrm{He}$ I 10830 line indicate that a complete dust shell formed around the object in late 1998-early 1999. Such a phenomenon also occurs in dust-forming classical novae.

The dust formation in V4334 Sgr and possibly in most massive final He flash objects is "catastrophic"; i.e., a shell is formed which surrounds the whole star and which does not dissipate quickly. V605 Aql, after its disappearance in 1924, never recovered from its dust episode: plates of the Sonneberg sky patrol from 1928-1979, reaching (mostly photographic) magnitudes of 16-17.5, did not recover it (Fuhrmann 1981). It was only recovered at a very faint magnitude (Seitter 1985). It is quite certain that V4334 Sgr will behave in a similar way in the years to come.

Thus, final He flash objects show some similarity to $\mathrm{R}$ CrB stars, but apparently the onset of R CrB-like activity, at least for the quickly evolving objects like V605 Aql and V4334 Sgr, soon ends in a catastrophic decline and does not extend over centuries of stellar evolution. The slowly evolv-

TABLE 5

Events In the Evolution of FG SAgittae, V605 Aquilae, aNd V4334 SagittariI

\begin{tabular}{|c|c|c|c|}
\hline Parameter & FG Sge & V605 Aql & V4334 Sge \\
\hline Brightness increase (spectrum) ................... & $1894-1975[\mathrm{~B}-\mathrm{G} 2 \mathrm{I}]$ & 1917.7-1918 & 1994.8-1995 \\
\hline Time of brightness maximum in $B$ (spectrum) ...... & $1968[\mathrm{~A} 3 \mathrm{I}]$ & 1919.6 & B $1996.3[\mathrm{~F} 0]$ \\
\hline Spectrum at later stage $\ldots \ldots \ldots \ldots \ldots \ldots \ldots \ldots \ldots$ & G-K0 $\mathrm{I}$ in $1980 \mathrm{~s}$ & $\mathrm{C} 2,2$ in 1921.7 & $\mathrm{C} 2,2$ in 1997.3 \\
\hline Onset of dust formation $\ldots \ldots \ldots \ldots \ldots \ldots \ldots \ldots \ldots$ & 1992 & $1922.6(?)$ & 1998.4 \\
\hline Dramatic decline ("disappearance")................ & $?$ & 1924 & 1999.2 \\
\hline
\end{tabular}


ing object FG Sge is also much more active than normal $\mathrm{R}$ $\mathrm{CrB}$ stars, but it has shown blue declines, indicating that only localized dust formation has occurred until now. It will be extremely interesting to follow the future behavior of FG Sge. Final He flash objects as we know them are obviously not settling down as "normal" R CrB stars. Possibly lowmass, very slowly evolving final He flash objects (with FG Sge possibly defining the high-mass limit) are the ones that may show up as R CrB stars during extended evolutionary phases.

The rapid evolution of V4334 Sgr (and of V605 Aql) indicate that we observe here the massive objects undergoing post-AGB evolution. Models covering such masses and timescales are badly needed in order to constrain masses, luminosities, and distances of the observed events.

This paper profited much from a stay of H. W. D. at the Space Telescope Science Institute in Baltimore. He thanks
M. Shara and N. Panagia for support and encouragement and K. Sahu for arranging a seminar talk. Helpful electronic discussions with M. Asplund (Uppsala), A. Evans (Keele), U. S. Kamath and N. M. Ashok (Ahmedabad), and Ya. Pavlenko (Kiev) are gratefully recognized, and we are also very much indebted to P. A. Whitelock (South African Astronomical Observatory) for communicating infrared data in advance of publication, to G. Wallerstein (Seattle) for communicating spectroscopic observations, and to W. C. Seitter for a careful reading of the manuscript. C. S. and T. A. acknowledge financial support from the Fund for Scientific Research Flanders. This research was supported by the Belgian Fund for Scientific Research and by the Flemish Ministry for Foreign Policy, European Affairs, Science, and Technology. Finally, we acknowledge the comments of a referee, which were very helpful in improving the presentation of the paper.
Acker, A., Fresneau, A., Pottasch, S. R., \& Jasniewicz, G. 1998, A\&A, 337, 253

Acker, A., Ochsenbein, F., Stenholm, B., Tylenda, R., Marcout, J., \& Schohn, C. 1992, Strasbourg-ESO Catalogue of Galactic Planetary Nebulae (Garching bei München: ESO)

Arkhipova, V. P., Esipov, V. F., Noskova, R. I., Sokol, G. V., Tatarnikov, A. M., Shenavrin, V. I., Yudin, B. F., Munari, U., \& Rejkuba, M. 1998, Astron. Lett., 24, 248

Arkhipova, V. P., \& Noskova, R. I. 1997, Astron. Lett., 23, 623

Arkhipova, V. P., Noskova, R. I., Esipov, V. F., \& Sokol, G. V. 1999, Astron. Lett., 25, 615

Asplund, M. 1998, A\&A, 330, 641

Asplund, M., Gustafsson, B., Lambert, D. L., \& Rao, N. K. 1997, A\&A, 321, L17

Barnbaum, C., Stone, R. P. S., \& Keenan, P. C. 1996, ApJS, 105, 419

Bath, G. T., \& Harkness, R. P. 1989, in Classical Novae, ed. M. F. Bode \& A. Evans (Chichester, NY: Wiley), 61

Bidelman, W. P. 1973, BAAS, 5, 442

Blöcker, T. 1995, A\&A, 299, 755

Blöcker, T., \& Schönberner, D. 1997, A\&A, 324, 991

Clayton, G. C., \& De Marco, O. 1997, AJ, 114, 2679

Cottrell, P. L., Lawson, W. A., \& Buchhorn, M. 1990, MNRAS, 244, 149

Duerbeck, H. W., \& Benetti, S. 1996, ApJ, 468, L111 (D96)

Duerbeck, H. W., Benetti, S., Gautschy, A., van Genderen, A., Kemper, C., Liller, W., \& Thomas, T. 1997, AJ, 114, 1657 (D97)

Duerbeck, H. W., \& Pollacco, D. 1996, IAU Circ. 6328

Duerbeck, H. W., et al. 1998, in Unsolved Problems in Stellar Evolution, ed. M. Livio (Cambridge: Cambridge Univ. Press), 17

Eyres, S. P. S., Evans, A., Geballe, T. R., Salama, A., \& Smalley, B. 1998a, MNRAS, 298, L37

Eyres, S. P. S., Richards, A. M. S., Evans, A., \& Bode, M. F. 1998b, MNRAS, 297, 905

Eyres, S. P. S., Smalley, B., Geballe, T. R., Evans, A. Asplund, M., \& Tyne, V. H. 1999, MNRAS, 307, L11

Feast, M. W., Carter, B. S., Roberts, G., Marang, F., \& Catchpole, R. M. 1997, MNRAS, 285, 317

Fernie, J. D., Percy, J. R., \& Richer, M. G. 1986, PASP, 98, 605

Forrest, W. J., Gillett, F. C., \& Stein, W. A. 1972, ApJ, 178, L129

Fuhrmann, B. 1981, Mitt. Veränderl. Sterne, 9, 13

Gallagher, J. S., \& Ney, E. P. 1976, ApJ, 204, L35

Gonzalez, G., Lambert, D. L., Wallerstein, G., Kameswara Rao, N., Smith, V. V., \& McCarthy, J. K. 1998, ApJS, 114, 133

Guinan, E. F., Dewarf, L. E., McCook, G. P., Dituro, P., Mittal, R., \& Margheim, S. J. 1998, BAAS, 193, 1510

Hakkila, J., Myers, J. M., Stidham, B. J., \& Hartmann, D. H. 1997, AJ, 114, 2043

Harrison, T. E. 1996, PASP, 108, 1112
Hinkle, K., \& Joyce, R. 1999, IAU Circ. 7266

Iben, I., Jr., \& MacDonald, J. 1995, in White Dwarfs, ed. D. Koester \& K. Werner (New York: Springer), 48

Jacoby, G. 1979, PASP, 91, 754

Jacoby, G., \& De Marco, O. 1998, IAU Circ. 7065

Jacoby, G., De Marco, O., \& Sawyer, D. G. 1998, AJ, 116, 1367

Jacoby, G. H., \& Ford, H. C. 1983, ApJ, 266, 298

Jurcsik, J., \& Montesinos, B. 1999, NewA Rev., 43, 415

Kaeufl, H. U., \& Stecklum, B. 1998, IAU Circ. 6938

Kamath, U. S., \& Ashok, N. M. 1999, MNRAS, 302, 512

Kerber, F., Blommaert, J. A. D. L., Groenewegen, M. A. T., Kimeswenger,

S., Käufl, H. U., \& Asplund, M. 1999, A\&A, 350, L27

Kimeswenger, S., Gratl, H., Kerber, F., Fouqué, P., Kohle, S., \& Steele, S. 1997, IAU Circ. 6608

Kimeswenger, S., \& Kerber, F. 1998, A\&A, 330, L41

Kipper, T. 1999, Inf. Bull. Variable Stars, 4707

Kipper, T., \& Klochkova, V. G. 1997, A\&A, 324, L65

Lawson, W. A., \& Cottrell, P. L. 1989, MNRAS, 240, 689

Lawson, W. A., Cottrell, P. L., Gilmore, A. C., \& Kilmartin, P. M. 1992, MNRAS, 256, 339

Lawson, W. A., Cottrell, P. L., Kilmartin, P. M., \& Gilmore, A. C. 1990, MNRAS, 247,91

Liller, W., Duerbeck, H. W., van der Meer, A., \& van Genderen, A. M. 1998b, IAU Circ. 7049

Liller, W., Janson, M., Duerbeck, H. W., \& van Genderen, A. M. 1998a, IAU Circ. 6825

Lynch, D. K., Russell, R. W., Rice, C. J., \& Sitko, M. 1998, IAU Circ. 6952

Margheim, S. J., Guinan, E. F., \& McCook, G. P. 1997, BAAS, 191, 4301

Mattei, J. A., Waagen, E. O., \& Foster, E. G. 1991, R Coronae Borealis

Light Curves: 1843-1990 (Cambridge: AAVSO)

Menzies, J. W., \& Feast, M. W. 1997, MNRAS, 285, 358

Nakano, S., Benetti, S., \& Duerbeck, H. W. 1996, IAU Circ. 6322

Ney, E. P., \& Hatfield, B. F. 1978, ApJ, 219, L111

Pavlenko, Ya. V., Yakovina, L. A., \& Duerbeck, H. W. 2000, A\&A, 354, 229

Pollacco, D. 1999, MNRAS, 304, 127

Querci, M., \& Querci, F. 1978, A\&A, 70, L45

Seitter, W. C. 1985, Mitt. Astron. Ges., 63, 181

Takamizawa, K. 1997, VSOLJ Variable Star Bull., 25, 4

Tyne, V. H., Eyres, S. P. S., Geballe, T. R., Evans, A., Smalley, B., Duerbeck, H. W., \& Asplund, M. 2000, MNRAS, submitted

van Genderen, A. M., \& Gautschy, A. 1995, A\&A, 294, 453

Werner, K., Dreizler, S., Rauch, T., Koesterke, L., \& Heber, U. 1999, in IAU Symp. 191, Asymptotic Giant Branch Stars, ed. T. Le Bertre, A. Lèbre, \& C. Waelkens (San Francisco: ASP), 493

Woitke, P., Goeres, A., \& Sedlmayer, E. 1996, A\&A, 313, 217 